\title{
KILOPARSEC-SCALE SIMULATIONS OF STAR FORMATION IN DISK GALAXIES. III. STRUCTURE AND DYNAMICS OF FILAMENTS AND CLUMPS IN GIANT MOLECULAR CLOUDS
}

\author{
Michael J. Butler ${ }^{1}$, Jonathan C. Tan ${ }^{2}$, and Sven Van Loo ${ }^{3,4}$ \\ ${ }^{1}$ Institute for Computational Science, University of Zurich, 8049 Zurich, Switzerland \\ ${ }^{2}$ Departments of Astronomy and Physics, University of Florida, Gainesville, FL 32611, USA \\ ${ }^{3}$ School of Physics and Astronomy, University of Leeds, Leeds LS2 9JT, UK \\ ${ }^{4}$ Harvard-Smithsonian Center for Astrophysics, 60 Garden Street, Cambridge, MA 02138, USA \\ Received 2014 October 18; accepted 2015 February 20; published 2015 May 13
}

\begin{abstract}
We present hydrodynamic simulations of self-gravitating dense gas in a galactic disk, exploring scales ranging from $1 \mathrm{kpc}$ down to $\sim 0.1 \mathrm{pc}$. Our primary goal is to understand how dense filaments form in giant molecular clouds (GMCs). These structures, often observed as infrared dark clouds (IRDCs) in the Galactic plane, are thought to be the precursors to massive stars and star clusters, so their formation may be the rate-limiting step controlling global star formation rates in galactic systems as described by the Kennicutt-Schmidt relation. Our study follows on from Van Loo et al., which carried out simulations to $0.5 \mathrm{pc}$ resolution and examined global aspects of the formation of dense gas clumps and the resulting star formation rate. Here, using our higher resolution, we examine the detailed structural, kinematic, and dynamical properties of dense filaments and clumps, including mass surface density $(\Sigma)$ probability distribution functions, filament mass per unit length and its dispersion, lateral $\Sigma$ profiles, filament fragmentation, filament velocity gradients and infall, and degree of filament and clump virialization. Where possible, these properties are compared to observations of IRDCs. By many metrics, especially too large mass fractions of high $\Sigma>1 \mathrm{~g} \mathrm{~cm}^{-2}$ material, too high mass per unit length dispersion due to dense clump formation, too high velocity gradients, and too high velocity dispersion for a given mass per unit length, the simulated filaments differ from observed IRDCs. We thus conclude that IRDCs do not form from global fast collapse of GMCs. Rather, we expect that IRDC formation and collapse are slowed significantly by the influence of dynamically important magnetic fields, which may thus play a crucial role in regulating galactic star formation rates.
\end{abstract}

Key words: galaxies: ISM - galaxies: star clusters: general - ISM: clouds - ISM: structure - methods: numerical stars: formation

\section{INTRODUCTION}

Stars form from molecular clouds in the interstellar medium (ISM), especially giant molecular clouds (GMCs; McKee \& Ostriker 2007 hereafter MO07; Tan et al. 2013). The rate of star formation appears to be relatively inefficient compared to that derived from collapse of the clouds at the free-fall rate (Zuckermann \& Evans 1974; Krumholz \& Tan 2007). Part of the reason for this may be the high degree of clustering of star formation within GMCs in regions with $A_{V} \gtrsim 10 \mathrm{mag}$ (Heiderman et al. 2010; Lada et al. 2010; Gutermuth et al. 2011). Studying the formation of the dense substructures within molecular clouds is thus crucial for a more complete understanding of the star formation process.

Observationally, these dense substructures have been studied by various molecular line tracers, such as ${ }^{13} \mathrm{CO}$ (e.g., Jackson et al. 2006), $\mathrm{HCO}^{+}$(e.g., Barnes et al. 2011), and $\mathrm{N}_{2} \mathrm{H}^{+}$(e.g., Henshaw et al. 2013); sub-mm and $\mathrm{mm}$ dust continuum emission (e.g., Rathborne et al. 2006; Ginsburg et al. 2012); and mid-infrared extinction (e.g., Butler \& Tan 2009, 2012; Peretto \& Fuller 2009) of infrared dark clouds (IRDCs).

Theoretically, we expect gravitational collapse within molecular clouds to be mediated by support from some combination of turbulence, magnetic fields, and stellar feedback (MO07). Some examples of recent work studying dense gas formation include setups of internal GMC converging flows (Chen \& Ostriker 2014), global turbulent clouds (Smith et al. 2014), periodic box turbulence (Moeckel \& Burkert 2014), and formation of GMCs from converging atomic flows (Heitsch et al. 2009; Gomez \& VázquezSemadeni 2014).

Our approach differs from these previous studies by setting the boundary conditions for GMCs from a galactic environment affected by global galactic dynamics. Tasker \& Tan (2009, hereafter TT09) carried out hydrodynamic simulations of an idealized axisymmetric, flat rotation curve galaxy to resolve the formation and interaction of GMCs (see also Dobbs 2008; Bournaud et al. 2010; Renaud et al. 2013). Their mutual interactions lead to a supersonic velocity dispersion of the clouds and frequent collisions that drive turbulence in the gas. In order to understand the star formation process within molecular clouds, a significant range of the cloud's internal structure then needs to be resolved, ranging from the GMCscale down to the $\sim 1 \mathrm{pc}$ scale clumps thought to represent the initial conditions of star cluster formation. Van Loo et al. (2013, hereafter Paper I) followed a $1 \mathrm{kpc}^{2}$ patch of the TT09 disk (extended vertically for $\pm 1 \mathrm{kpc}$ ) down to a resolution of $0.5 \mathrm{pc}$ for a period of $10 \mathrm{Myr}$. Star formation was introduced in gas above a threshold "clump" density of $n_{\mathrm{H}}=10^{5} \mathrm{~cm}^{-3}$ and at a star formation efficiency per local free-fall time of $\epsilon_{\mathrm{ff}}=0.02$. The star particles created, with a minimum mass of $100 M_{\odot}$ representing clusters or subclusters of stars, were prevented from accreting additional gas. Nor was local feedback from these star particles implemented. In spite of the relatively low value of $\epsilon_{\mathrm{ff}}$, the overall SFR seen in the simulation was much ( $\sim 100$ times) larger than in observed galaxies with similar total mass surface densities of gas (e.g., 
Bigiel et al. 2008). This was due in part to the much higher mass fractions of gas at "GMC" and "clump" densities than in real galaxies: about $70 \%$ of the gas was within "GMCs" (at $n_{\mathrm{H}} \geqslant 10^{2} \mathrm{~cm}^{-3}$ ), and of this about $50 \%$ was also above the clump threshold density. In Paper I, we speculated that inclusion of magnetic fields and/or local feedback from young stars is needed to resolve this discrepancy.

In Van Loo et al. (2015, Paper II) we presented an initial study of the effects of magnetic fields of various strengths on the same kpc-scale patch of the galactic disk, finding modest levels of global suppression of star formation rates by up to factors of two. However, this result was strongly influenced by the presence of a single magnetically supercritical starburst region in one part of the simulation domain, and larger suppression factors were seen in other regions.

Our goal in this paper, Paper III, is to follow the evolution of the GMCs and the formation of dense filaments and clumps to higher spatial resolution, $0.122 \mathrm{pc}$. This is carried out with the same physics as modeled in Paper I, namely, pure hydrodynamics of self-gravitating gas (magnetic fields at this resolution and stellar feedback are deferred to future papers). The rationale is to be able to carry out more detailed characterization of the stucture, kinematics, and dynamics of forming dense gas structures for comparison with Galactic IRDCs, which, being at very early stages of their star formation, are probably relatively unaffected by local stellar feedback. Our particular focus is on the properties of long $(\sim 50 \mathrm{pc})$ filaments that form from the collapsing GMCs. We measure various properties of simulated filaments and compare to similarly long filaments, recently discovered, including as IRDCs, in the Galactic ISM (e.g., Jackson et al. 2010; Battersby \& Bally 2012; Ragan et al. 2014).

These nonmagnetic, zero-feedback simulations should thus be regarded as baseline calculations from which we can then determine how much, if any, suppression of collapse is needed from magnetic fields (and stellar feedback) to more accurately represent the observed structure and dynamics of dense, starforming filaments and clumps.

In Section 2 we describe our methods and numerical setup. In Sections 3 and 4 we describe our results. In Section 5 we conclude.

\section{METHODS AND NUMERICAL SETUP}

For our initial conditions, we use the same $1 \mathrm{kpc}^{2}$ patch $(x-y$ coordinates describe location in the disk plane) studied in Paper I, that is, centered at a galactocentric radius of $4.25 \mathrm{kpc}$ (the galactic center is $4.25 \mathrm{kpc}$ away from the patch center in the negative $x$ direction and at the same $y$ value) and extending to $z= \pm 1 \mathrm{kpc}$, above and below the disk. This patch was extracted from the global galaxy simulation of TT09 after a time when the disk was largely fragmented into a population of GMCs (see left column of Figure 1). As in Paper I, the velocity field is transformed to the frame of the circular velocity of $200 \mathrm{~km} \mathrm{~s}^{-1}$ at the center of the box. Periodic boundary conditions are applied at the $x-z$ faces of box and outflow boundary conditions at the other faces. A fixed background potential is applied to represent the vertical distribution of galactic stars and dark matter to match the potential used by TT09. Note that this setup is not that of a shearing box (e.g., Coriolis forces are neglected), but the effects of this approximation are expected to be quite small since the total time span that is followed is relatively short compared to a flow crossing time across the box.

Paper I followed the evolution of this region for $10 \mathrm{Myr}$ with a maximum resolution of $0.49 \mathrm{pc}$, but, to resolve structures on the scale of individual star-forming clumps, higher resolution is needed. The following simulations contain six levels of AMR on top of the original $7.8 \mathrm{pc}$ base grid resolution of the TT09 simulation. The maximum resolution is then $0.122 \mathrm{pc}$, i.e., four times better than in Paper I.

We include heating and cooling functions derived using the photodissociation code Cloudy (version 8; Ferland et al. 1998), as in Paper I. These functions are able to treat gas at temperatures as low as $5 \mathrm{~K}$ (and up to $\sim 10^{5} \mathrm{~K}$ and beyond). These functions include both atomic and molecular line cooling processes, including from $\mathrm{H}_{2}$ and $\mathrm{CO}$, among others. A table of heating and cooling rates for a range of densities and temperatures was generated based on the density versus mean extinction relationship derived in Paper I. For temperatures above $T=10^{5} \mathrm{~K}$, we opt to use the cooling curve of Sarazin \& White (1987) and set the heating rate to zero. For densities and temperatures above or below the limits of the table, we use the limiting rate. For more details on the derivation of this function, see Paper I.

Since our focus is on the dense, molecular gas, we adopt a fixed mean particle mass of $\mu=2.33 m_{\mathrm{H}}=3.90 \times 10^{-24} \mathrm{~g}$, i.e., assuming $n_{\mathrm{He}}=0.1 n_{\mathrm{H}}$. Thus, the sound speed is $c_{\text {th }}=(\gamma P / \rho)^{1 / 2}$, which implies an adiabatic sound speed $c_{\text {th }}=(5 k T /[3 \mu])^{1 / 2} \rightarrow 0.243(T / 10 \mathrm{~K})^{1 / 2} \mathrm{~km} \mathrm{~s}^{-1}$. Our use of this fixed value of $\mu$ means that the pressures of the regions of our simulation that correspond to atomic regions are smaller in our simulation by a factor of 1.83 than they would be in reality.

We present the results of two separate simulation runs. The first run, Run $\mathrm{nSF}$, includes all the above processes at a maximum resolution of $0.122 \mathrm{pc}$. In the second run, Run SF, we utilize a simple recipe for star formation, which converts a fixed percentage of gas mass per free-fall time into star particles if a cell exceeds a particular threshold density. We choose a value for the star formation efficiency per local free-fall time, $\epsilon_{\mathrm{ff}}=0.02$ (Krumholz \& Tan 2007). As in Paper I, we do not resolve individual star-forming cores, so no requirements for the gas to be converging or to be gravitationally bound are imposed. When a cell exceeds the threshold density, a star particle is formed whose mass is calculated by

$$
M_{*}=\epsilon_{\mathrm{ff}} \frac{\rho \Delta x^{3}}{t_{\mathrm{ff}}} \Delta t,
$$

where $\rho$ is the gas density, $\Delta x^{3}$ the cell volume, $\Delta t$ the numerical time step, and $t_{\mathrm{ff}}$ the free-fall time of gas in the cell (evaluated as $t_{\mathrm{ff}}=(3 \pi / 32 G \rho)^{1 / 2}$ with a mean molecular weight of $\mu=2.33$ ). We use a threshold density of $n_{\mathrm{H}}=10^{6} \mathrm{~cm}^{-3}$ and a minimum star particle mass of $10 M_{\odot}$. If $M_{*}<M_{\min }$, then a particle is formed stochastically with a probability $M_{*} / M_{\min }$. At the threshold density, the minimum gas mass in the cell is $M_{* \text {,min }}=63 M_{\odot}$. Note that the threshold density is higher and minimum star particle mass smaller compared to Paper I, because of the higher grid resolution. The star particles are treated as collisionless particles whose motions are governed by pure $N$-body calculations, and do not gain any mass once they are formed. 


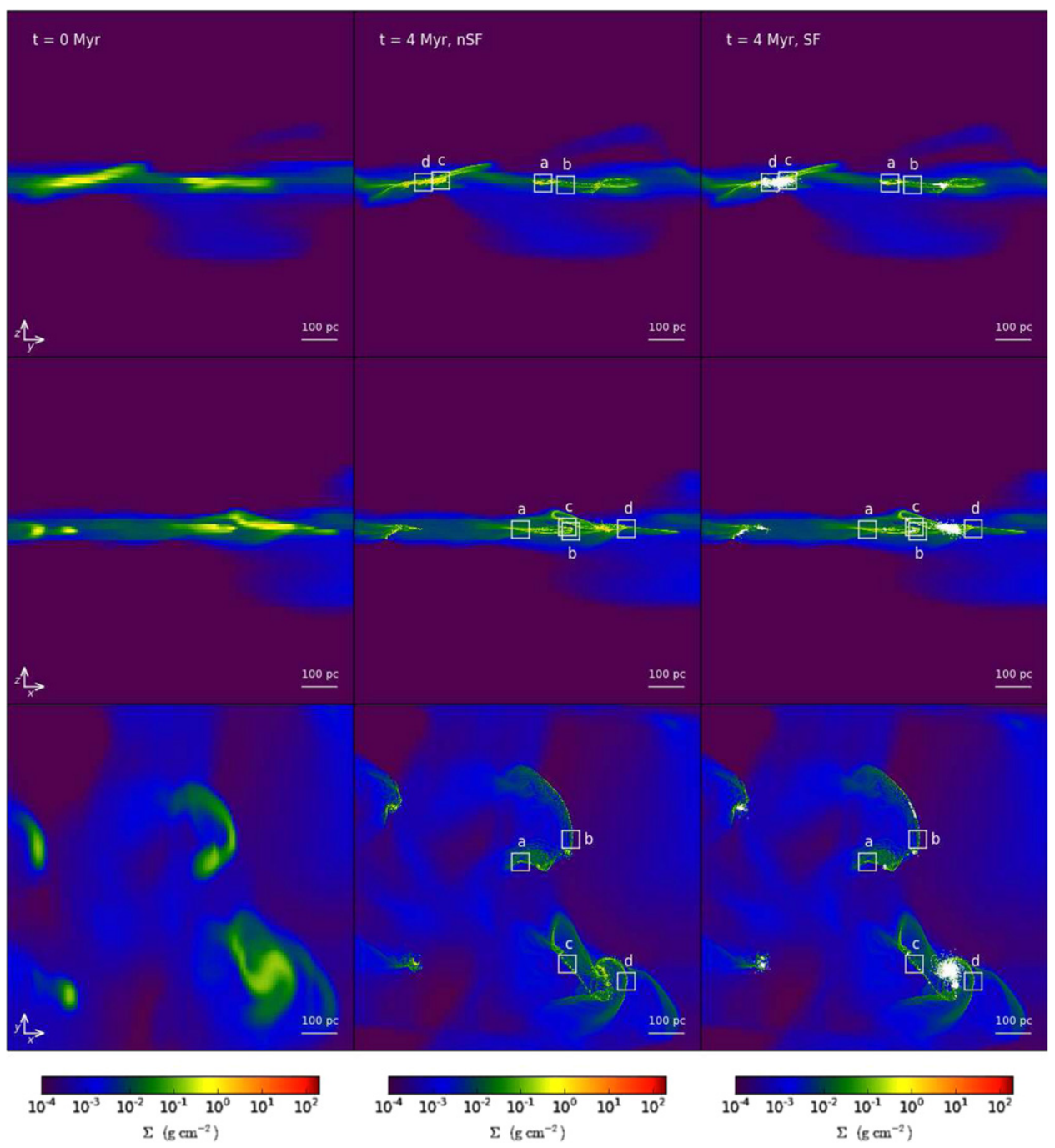

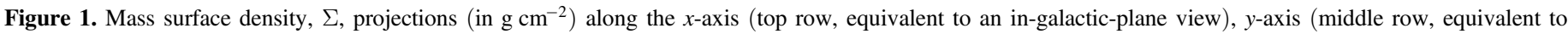

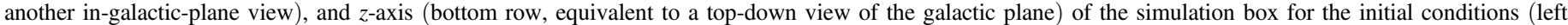

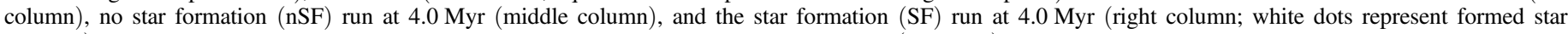

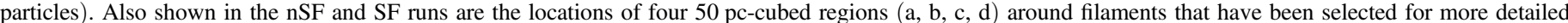
analysis (see text). We note that this figure is displayed at reduced resolution.

The focus of this study is the collapse of GMCs to form filaments and the fragmentation of these filaments to form dense star-forming clumps. Considering the timescales on which these processes occur, we run the simulations for $4 \mathrm{Myr}$ using the adaptive mesh refinement hydrodynamics code Enzo 2.0 (Bryan et al. 2014; O'Shea et al. 2004). After this time, the clouds have undergone significant fragmentation and formed a large number of small pc-scale clumps in several filaments (see Figure 1). In the run with star formation a large number of star particles has been created. The presence of a large number of dense gas and stellar structures makes continued calculation of the simulation very memory intensive, slow, and inefficient. Since continued evolution is not necessary for us to achieve our scientific goals of studying the initial stages of star formation and since other physics due to star formation feedback is not yet included in these models, we do not attempt to follow the evolution beyond $4 \mathrm{Myr}$.

We note that the Zeus solver (Stone \& Norman 1992), rather than a Godunov solver, has been used for these simulations. This introduces relatively large heating rates due to numerical viscosity, but makes the calculation more numerically stable. We have also carried out simulation runs at the $0.5 \mathrm{pc}$ resolution with this same solver for comparison.

\section{GLOBAL PROPERTIES OF THE ISM AND STAR FORMATION}

Figure 1 shows the mass surface density, $\Sigma$, of the initial conditions (left column) and after 4 Myr of evolution of Run nSF (middle column) and Run SF (right column) as seen along the cardinal axes. The mean locations of the GMCs do not change much over this relatively short time span, given the clouds' initial simulation-frame $x$-y-plane velocities of $\sim 20 \mathrm{~km} \mathrm{~s}^{-1}$ that are a mixture of the shearing velocity field of galactic rotation and the peculiar motions imparted by gravitational scattering interactions from prior evolution in the TT09 simulation. Note that these motions correspond to Mach numbers of about 100 for gas that has been able to cool to $\sim 10 \mathrm{~K}$.

After $4 \mathrm{Myr}$, the clouds are seen to have collapsed to form filamentary structures and more spheroidal clumps. The overall 


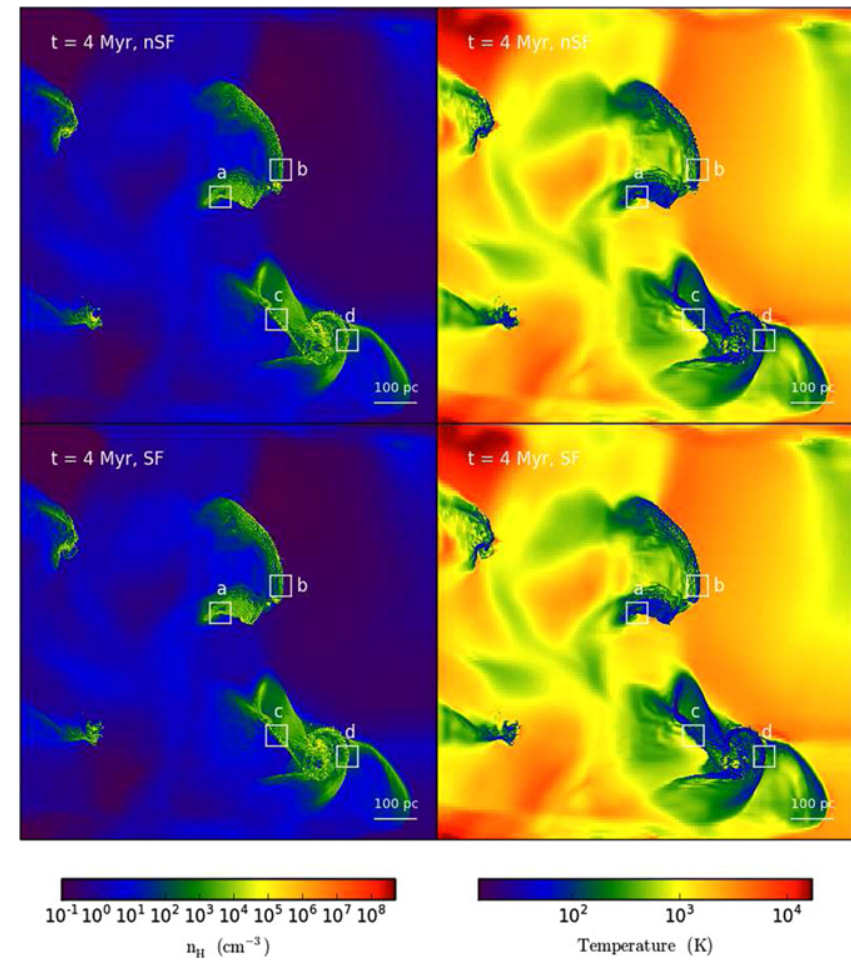

Figure 2. Mass-weighted mean density, $n_{\mathrm{H}}$ (left column), and temperature, $T$ (right column), of the top-down views of the nSF (top row) and SF (bottom row) run simulations at $4 \mathrm{Myr}$. We note that this figure is displayed at reduced resolution.

morphology of these gas structures is very similar between the $\mathrm{nSF}$ and SF runs. The main difference is seen in the peak $\Sigma$ values, with the SF run having lower values due to conversion of gas into star particles. The peak $\Sigma$ values as seen in the topdown projections are 1250 and $317 \mathrm{~g} \mathrm{~cm}^{-2}$ in the nSF and SF runs, respectively. Similarly, the peak gas density is $n_{\mathrm{H}}=8.4 \times 10^{8} \mathrm{~cm}^{-3}$ and $n_{\mathrm{H}}=4.1 \times 10^{8} \mathrm{~cm}^{-3}$ in these runs.

Figure 2 shows the mass-averaged mean densities, $n_{\mathrm{H}}$ (assuming $n_{\mathrm{He}}=0.1 n_{\mathrm{H}}$ ), and temperatures, $T$, of the $\mathrm{nSF}$ and $\mathrm{SF}$ runs at $4 \mathrm{Myr}$. The GMC-like structures have densities $\gtrsim 100 \mathrm{~cm}^{-3}$ and are often, but not always, embedded in relatively dense $\mathrm{H}_{\mathrm{I}}$ structures with $n_{\mathrm{H}} \sim 10 \mathrm{~cm}^{-3}$ and temperatures of $\sim 100-1000 \mathrm{~K}$. There are some cases, e.g., the GMC containing filament $\mathrm{b}$, where the dense molecular gas is moving into relatively low density, warmer gas. We note that since ionizing photon, stellar wind, and supernova (SN) feedback are not included, the global density and thermal structure of this simulated ISM lacks the hot phase component and underrepresents the warm phase components.

The total gas mass in Run nSF is $1.67 \times 10^{7} M_{\odot}$, while it falls by $\sim 10 \%$ to $1.50 \times 10^{7} M_{\odot}$ in Run SF after 4 Myr due to star formation activity. As a consequence, the mass in star particles grows to $1.67 \times 10^{6} M_{\odot}$, composed of $1.82 \times 10^{5}$ particles, resulting in a mean star particle mass of $19.2 M_{\odot}$. This means that, contrary to Paper I, not all star particles are formed stochastically at the minimum mass level. In fact, only a small percentage, $1.5 \%$, has the minimum star particle mass of $10 M_{\odot}$. The maximum star particle mass in our simulation is $289 M_{\odot}$. The average star formation rate per unit area during the 4.0 Myr of the simulation is $0.76 M_{\odot} \mathrm{yr}^{-1} \mathrm{kpc}^{-2}$.

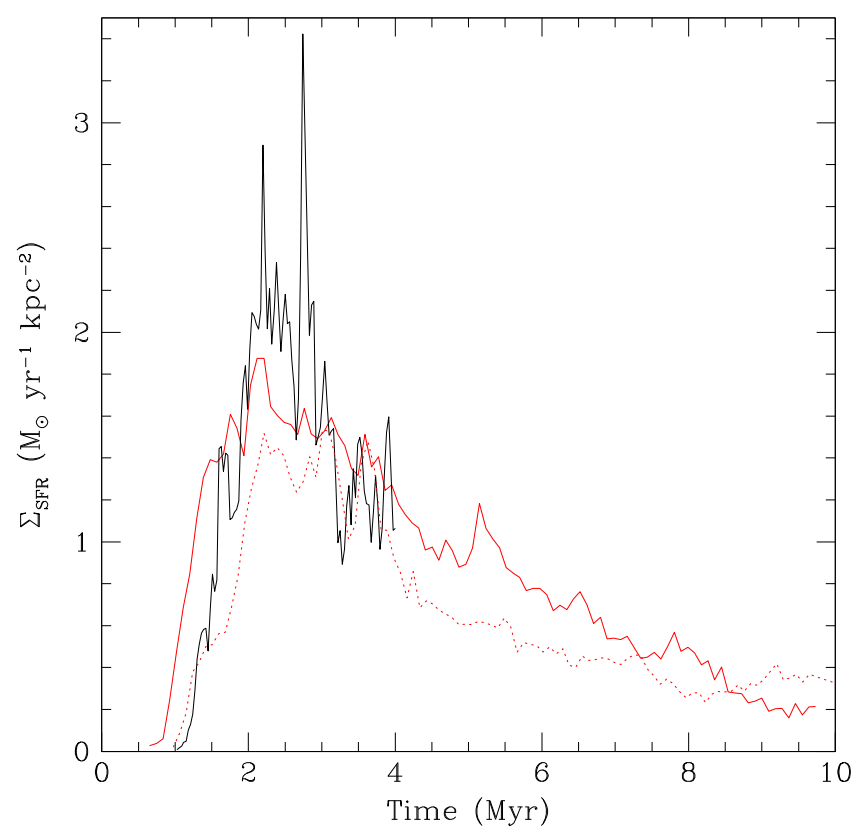

Figure 3. Time evolution of star formation rate per unit area for the $0.1 \mathrm{pc}$ resolution Run SF (black solid line), the 0.5 pc-resolution results from Paper I using the Godunov solver (red solid line), and a 0.5 pc-resolution result using the Zeus solver (red dotted line).

In Figure 3, we compare the SFR time evolution of Run SF to those of the $0.5 \mathrm{pc}$ resolution runs including star formation with a density threshold of $n_{\mathrm{H}}=10^{5} \mathrm{~cm}^{-3}$ and a minimum star particle mass of $100 M_{\odot}$ (equivalent to the simulations of Paper I, but also investigating the effect of the use of the Zeus rather than the Godunov solver). In all cases the evolution is characterized by an onset of star formation after $\sim 1 \mathrm{Myr}$, rising to a peak at $\sim 2-3 \mathrm{Myr}$, followed by a gradual decline. This evolution should be viewed as a response to the initial conditions of the simulation setup, where dense, self-gravitating gas clouds are released synchronously at $t=0$ and allowed to collapse to high densities to form stars. After an initial burst of star formation, the rate is seen to decline by factors of about 5 after 10 Myr. The choice of hydrodynamics solver is seen to make a 10\%-20\% difference to the SFR, with the additional heating introduced by the Zeus solver reducing the SFR and delaying its onset and peak. The higher resolution of Run SF, which involves a higher threshold density for star formation, leads to increased and more time-variable SFRs compared to the lower-resolution run that also uses the Zeus solver.

Similar to the results of Paper I, the overall SFRs per unit area are much higher, by factors of $\sim 100$, than in observed galaxies (e.g., Bigiel et al. 2008), most likely due to effects of magnetic fields and/or feedback from newly formed stars that are present in real galaxies, but lacking in these simulations. The quantitative effect of magnetic fields for the same simulation setup as Paper I has been investigated by Van Loo et al. (2015).

\section{FILAMENT STRUCTURE, KINEMATICS, AND DYNAMICS}

\subsection{Filament Selection and Bulk Environmental Properties}

We select a sample of four large filaments, a-d, from the simulated GMCs at 4.0 Myr from the SF run for a detailed study of their structure, kinematics, and dynamics. These 
Table 1

Filament 50 and 25 pc Region Properties ${ }^{\mathrm{a}}$

\begin{tabular}{|c|c|c|c|c|c|c|c|}
\hline Filament & $\begin{array}{c}x_{c}, y_{c}, z_{c} \\
(\mathrm{pc})\end{array}$ & $\begin{array}{c}M_{g} \\
\left(10^{4} M_{\odot}\right)\end{array}$ & $\begin{array}{c}n_{\mathrm{H}} \\
\bar{n}_{\mathrm{H}} \\
\left(10^{4} \mathrm{~cm}^{-3}\right)\end{array}$ & $\begin{array}{cl}f_{\Sigma 0.1, x} & f_{\Sigma 0.1, y} \\
f_{\Sigma 0.1, x} & f_{\bar{f}_{\Sigma 0.1,1, y}} \\
& \bar{\delta}_{\Sigma 0.1, z} \\
\left(10^{-2}\right)\end{array}$ & $\begin{array}{c}f_{\Sigma 1, x} f_{\Sigma 1, y} \\
\bar{f}_{\Sigma 1, x} \\
\bar{\Sigma}_{\Sigma 1, y} \\
\left(10^{-2}\right)\end{array}$ & $\begin{array}{l}v_{x}, v_{y}, v_{z} \\
\bar{v}_{x} \bar{y}_{y} \bar{z}_{z} \\
\left(\mathrm{~km} \mathrm{~s}^{-1}\right)\end{array}$ & $\begin{array}{c}\sigma_{v, x} \sigma_{v, y} \sigma_{v, z} \\
\bar{\sigma}_{v, x} \bar{\sigma}_{\bar{v}, y} \bar{\sigma}_{\bar{v}, z} \\
\left(\mathrm{~km} \mathrm{~s}^{-1}\right)\end{array}$ \\
\hline a & $480[482], 545[546], 3.00[1.38]$ & $22.3[12.4]$ & $\begin{array}{c}0.00517[0.0124] \\
145[259]\end{array}$ & $\begin{array}{c}3.8[4.9], 5.0[6.1], 1.4[3.5] \\
11[67], 38[22], 3.0[13]\end{array}$ & $\begin{array}{c}0.26[0.76], 0.088[0.29], 0.079[0.26] \\
0.80[7.3], 0.75[1.2], 0.271[0.42]\end{array}$ & $\begin{array}{l}-22.6[-24.4], 2.94[3.30],-1.81[-1.74] \\
-20.7[-20.9], 7.62[9.18],-1.87[-2.00]\end{array}$ & $\begin{array}{l}5.37[5.09], 6.54[7.86], 3.18[3.90] \\
5.75[5.29], 7.20[8.41], 5.62[7.39]\end{array}$ \\
\hline b & $625[626], 610[612],-3.50[-1.05]$ & $14.2[5.20]$ & $\begin{array}{l}0.00329[0.00964] \\
42.7[13.1]\end{array}$ & $\begin{array}{c}0.87[2.0], 1.9[4.0], 0.94[2.0] \\
8.0[36], 5.8[21], 0.99[9.0]\end{array}$ & $\begin{array}{l}0.14[0.25], 0.21[0.29], 0.14[0.27] \\
6.9[0.36], 0.014[0.015], 0.26[1.5]\end{array}$ & $\begin{array}{c}-9.05[-8.49],-10.3[-9.99],-1.01[-1.12] \\
-7.44[-6.56],-9.97[-9.89],-0.561[-0.519]\end{array}$ & $\begin{array}{l}5.93[5.63], 3.85[2.60], 2.58[2.46] \\
4.53[3.35], 4.31[2.50], 3.61[2.65]\end{array}$ \\
\hline$c^{\mathrm{b}}$ & $614[618], 251[248], 20.3[19.1]$ & $38.6[17.3]$ & $\begin{array}{l}0.00895[0.0321] \\
326[268]\end{array}$ & $\begin{array}{c}2.4[4.0], 3.7[7.3], 2.5[4.9] \\
39[48], 30[13], 28[56]\end{array}$ & $\begin{array}{c}0.41[0.85], 0.47[1.2], 0.42[0.86] \\
29[36], 7.9[2.0], 20[7.7]\end{array}$ & $\begin{array}{c}-20.4[-18.9],-11.6[-12.3],-0.433[-0.370] \\
-13.9[-13.5],-7.69[-8.81],-1.17[-1.35]\end{array}$ & $\begin{array}{l}7.35[7.78], 6.21[5.63], 4.37[4.17] \\
8.90[9.19], 6.80[4.50], 6.48[8.07]\end{array}$ \\
\hline$d$ & $785[783], 200[202], 10.0[5.98]$ & $20.0[8.63]$ & $\begin{array}{c}0.00464[0.0160] \\
44.9[59.6]\end{array}$ & $\begin{array}{c}3.5[4.4], 2.9[4.0], 2.0[3.3] \\
30[62], 16[20], 1.3[4.6]\end{array}$ & $\begin{array}{l}0.11[0.17], 0.26[0.39], 0.13[0.23] \\
0.95[0.26], 0.32[0.37], 0.14[0.25]\end{array}$ & $\begin{array}{l}-22.7[-21.4], 7.17[7.74], 1.23[1.16] \\
-18.3[-16.5], 11.7[13.5], 1.41[1.62]\end{array}$ & $\begin{array}{l}7.50[7.48], 8.87[8.00], 2.85[2.83] \\
7.06[6.15], 6.82[5.59], 4.02[4.30]\end{array}$ \\
\hline
\end{tabular}

Notes.

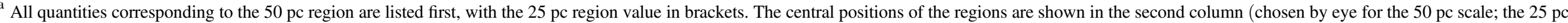

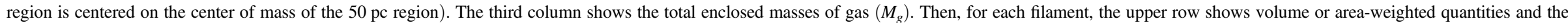

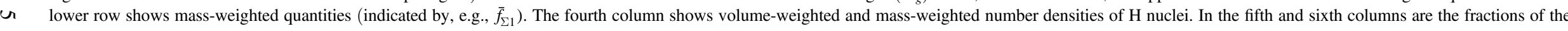
regions with $\Sigma>0.1$ and $1 \mathrm{~g} \mathrm{~cm}^{-2}$, respectively, as viewed along the $x-, y$-, and $z$ - axes. The seventh and eighth columns show the mean velocities and velocity dispersions, respectively.

Only this region has formed stars, with a total mass of $731 M_{\odot}$ 


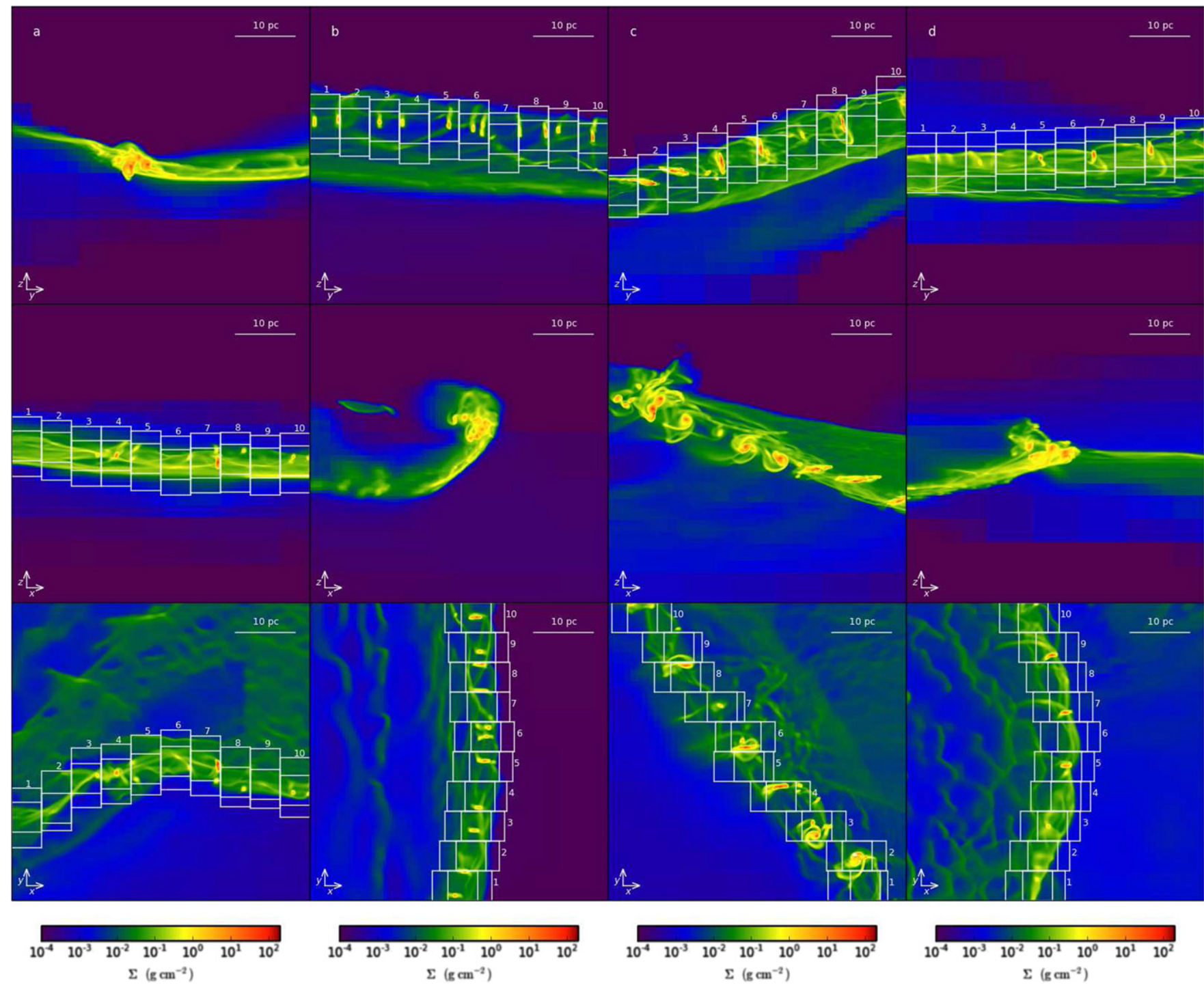

Figure 4. Mass surface density, $\Sigma$, projections (in $\mathrm{g} \mathrm{cm}^{-2}$ ) along the (top to bottom) $x$-, $y$-, and $z$-axes centered on Filaments a-d for the SF run at 4.0 Myr.

filaments are chosen to sample a variety of environments and star formation activities. Filaments $\mathrm{a}, \mathrm{b}$, and $\mathrm{d}$ are still undergoing fragmentation and collapse and have a lower level of star formation (it is expected to increase as they continue to collapse), while filament $\mathrm{c}$ is forming stars actively. The average mass in these $50 \mathrm{pc}$ scale filament regions is $\gtrsim 10^{5} \mathrm{M}_{\odot}$ (see Table 1). While this is similar to the typical mass of a massive Galactic GMC, note that these selected regions are just small parts of much larger and more massive molecular clouds/ complexes.

Mass surface density maps, with in-plane and top-down views, of the $50 \mathrm{pc}$-cubed regions containing the filaments are shown in Figure 4. The detailed structure of various degrees of fragmentation in the filaments can be seen, along with the structure of surrounding, more diffuse gas. The filaments tend to lie in directions parallel to the galactic plane, with filament $\mathrm{c}$ showing the largest deviation from an in-plane orientation.

Figure 4 also shows the division of the filaments into 10 "strips" (to be used for quantitative analysis of filament properties, below), each of $5 \mathrm{pc}$ width along the filament (chosen to be $x$-direction for filament a; $y$-direction for $\mathrm{b}, \mathrm{c}, \mathrm{d}$ ) and $10 \mathrm{pc}$ length perpendicular to the filament in each of the two directions of this orthogonal plane. The central positions of the strips in this plane are allowed to vary in order to track the filament, with the position located by first centering on the center of mass through the whole $50 \mathrm{pc}$ region as viewed in the $\Sigma$ projections, and then re-centering on the center of mass within that strip.

The volume densities, $n_{\mathrm{H}}$, mass-averaged through the $50 \mathrm{pc}$ filament regions, are shown in Figure 5. A wide range of densities, from $\sim 10^{-1}$ to $\sim 10^{7} \mathrm{~cm}^{-3}$, are present. Note that, lacking wind, ionization, and $\mathrm{SN}$ feedback, these simulations create this range of densities purely via gravitational collapse, diffuse FUV heating, and shocks resulting from GMC motions and interactions.

The mass-average mean simulation-frame velocities of the material in the $50 \mathrm{pc}$ regions are typically $\sim 20 \mathrm{~km} \mathrm{~s}^{-1}$ (see Table 1). The local velocity fields with respect to these region velocities are also shown in Figure 5, with typical values of $\sim 10 \mathrm{~km} \mathrm{~s}^{-1}$. Some large-scale converging flows are seen around the dense gas structures, together with other more disordered flows.

The mass-weighted temperatures of the gas along the various sight lines of the regions are shown in Figure 6. Again a wide 


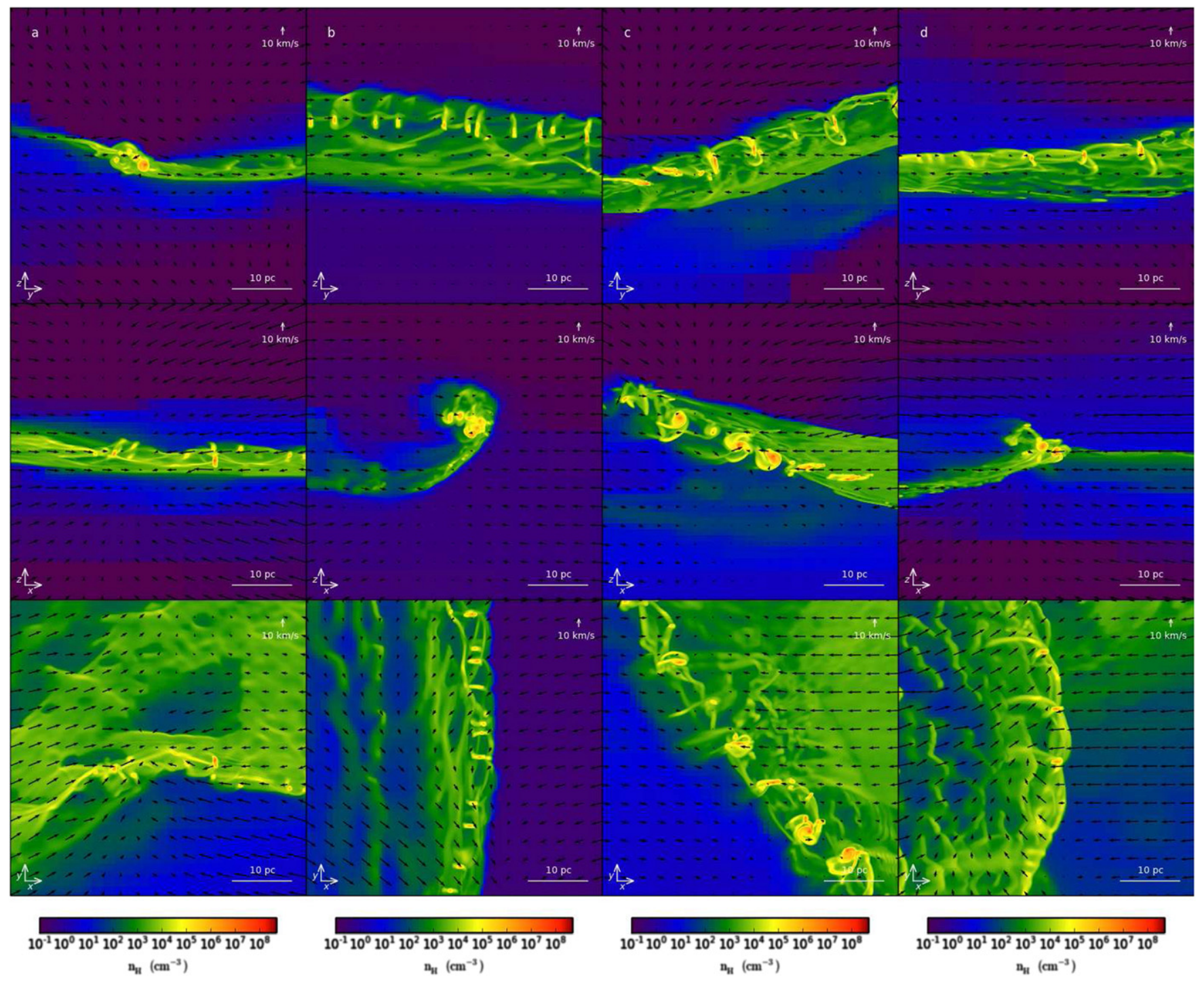

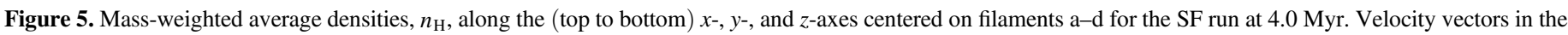
frame of the center of mass of the region are overplotted in black.

range of values are present, with the densest gas able to cool to $\lesssim 10 \mathrm{~K}$ and low-density regions reaching $\sim 10^{4} \mathrm{~K}$ from heating from the diffuse FUV radiation field and much higher temperatures $\left(\sim 5 \times 10^{6} \mathrm{~K}\right)$ from shocks, with speeds of $\sim 10-30 \mathrm{~km} \mathrm{~s}^{-1}$.

The probability distribution function (PDF) of mass surface density (or equivalently column density) can provide useful insight into the processes governing a cloud's structure. Kainulainen et al. (2009) showed that clouds undergoing star formation have wider column density PDFs, including a powerlaw tail of high column densities, while non-star-forming clouds have narrower distributions, better fit by a single lognormal function. The formation of PDF power-law tails has been interpreted as being due to evolution of a separate selfgravitating component of the clouds that are undergoing freefall collapse (e.g., Ballesteros-Paredes et al. 2011; Kritsuk et al. 2011). In this case the development of such PDF tails would mark the onset of star formation.

For each of the four filament regions in the SF run, we construct the mass-weighted $\Sigma$ PDF for the $50 \mathrm{pc}$ cube as viewed in each of the three orthogonal axes, $x, y, z$, and these are presented as the blue lines in Figure 7. A fair amount of structure can be present in the PDFs at all $\Sigma$ values, which we expect is partly a consequence of the global cloud structure that happens to be within the defined region. At the high- $\Sigma$ end of the distributions, power-law tails are often present but can show variation in their properties depending on the viewing angle.

We also zoom into a $25 \mathrm{pc}$ cube, centered on the center of mass of the regions, and show the $\Sigma$ PDFs of these regions (red lines in Figure 7). The differences compared to the $50 \mathrm{pc}$ cube regions illustrate the effect that the boundary region definition has on the PDF, including the normalization level of the powerlaw tails, which rise as one zooms in on the denser region.

Observationally, $\Sigma$ PDFs can be measured in a number of ways, including via sub-mm dust emission (which requires also knowing the temperature structure of the clouds and dust emissivity properties per unit total mass) and via near-IR (NIR) or mid-IT (MIR) extinction (which requires knowing dust opacities per unit total mass, but not cloud temperatures). In Figure 7 we show the $\Sigma$ PDF of IRDC C from the BT09 sample 


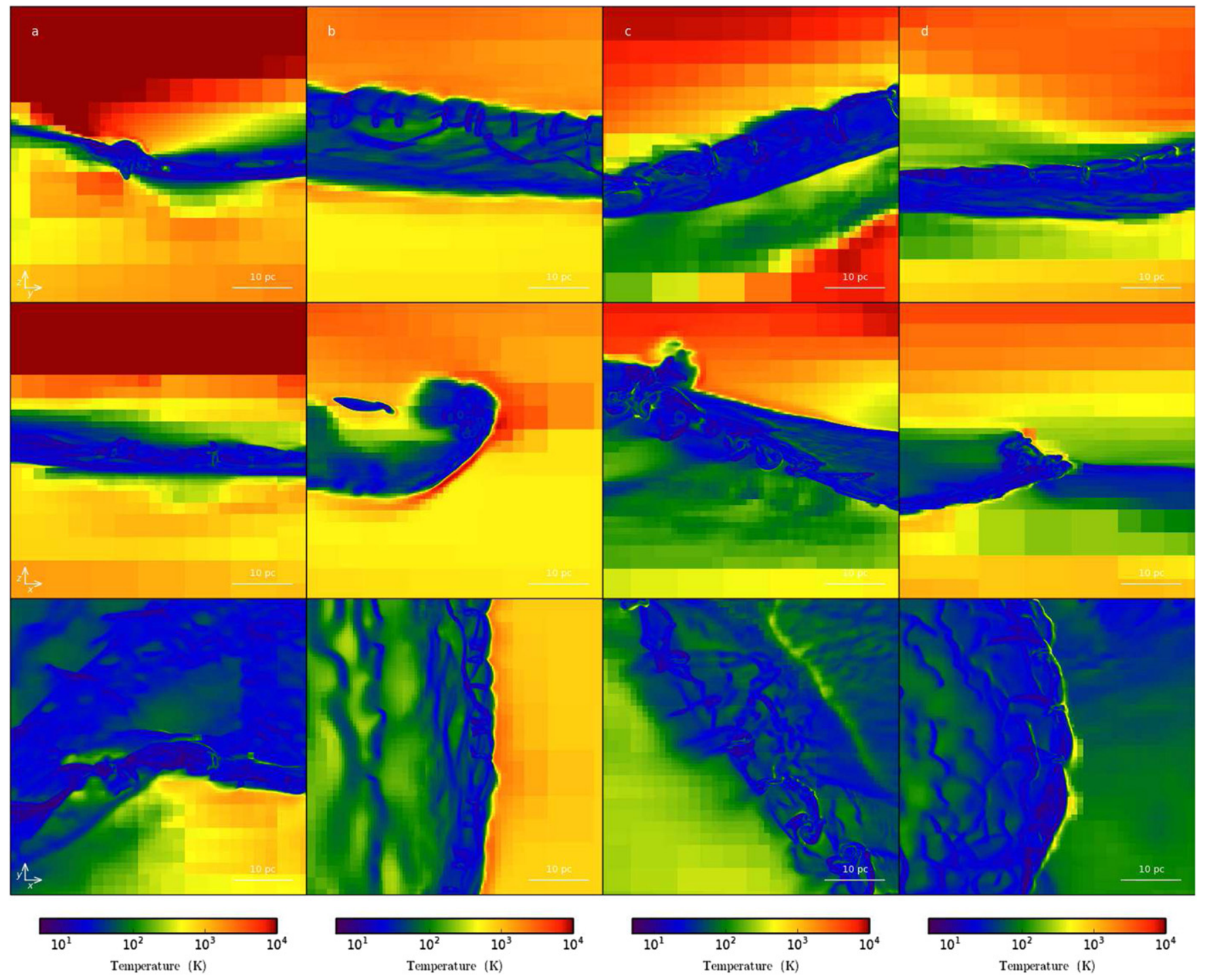

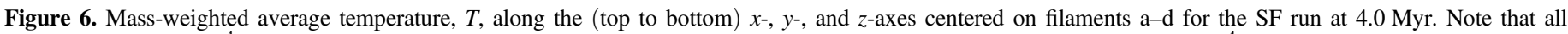

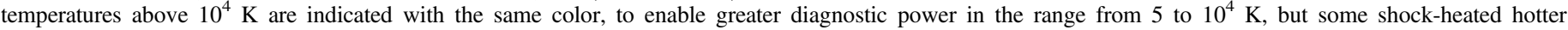
components are present up to $\sim 5 \times 10^{6} \mathrm{~K}$ (see text).

(G028.37+00.07) as measured in the study of Butler et al. (2014). This is one of the most massive and highest column density IRDCs known in the Galaxy. The PDF has been measured in a region that is designed to be complete for $A_{V} \gtrsim 3$ mag (i.e., $\Sigma \gtrsim 0.015 \mathrm{~g} \mathrm{~cm}^{-2}$ ), extending about $20^{\prime}$ on a side (i.e., about $29 \mathrm{pc}$ at the IRDC distance of $5 \mathrm{kpc}$ ). Note that the MIR extinction mapping technique has an upper limit of $\Sigma$ that it is able to probe, corresponding to $\sim 0.6 \mathrm{~g} \mathrm{~cm}^{-2}$. This region can be probed by sub-mm emission studies (e.g., Battersby et al. 2011; Schneider et al. 2014), but these results are dependent on the accuracy of the derived dust temperature and assumed emissivity properties, and they also have lower spatial resolution compared to the MIR extinction maps.

Allowing for the inability of the MIR extinction map to probe to very high $\Sigma$ values, the overall comparison is quite favorable. However, it should be noted that IRDC C is one of the more extreme examples known, while the filament regions selected in the simulation are quite moderate examples of dense regions. More typical IRDCs, selected from the BT12 sample, have been studied by Kainulainen \& Tan (2013), and these have relatively smaller amounts of gas at higher $\Sigma$ values. The GMCs and nearby star-forming regions studied by Kainulainen et al. (2009) show even smaller high- $\Sigma$ mass fractions. The largest values of $\Sigma$ that are seen within the kpc-scale volume of the simulations are $>100 \mathrm{~g} \mathrm{~cm}^{-2}$, much higher than have been seen via mm dust emission in IRDCs or star-forming clouds.

We evaluate the area and mass fractions of the PDFs that are $>0.1$ and $>1 \mathrm{~g} \mathrm{~cm}^{-2}$ and list the results in Table 1. There can be a large variation depending on orientation, i.e., if the viewing direction is perpendicular or parallel to the main axis of the filament. Again these values appear to be relatively high compared to observed IRDCs (e.g., KT13, BTK14), although care must be taken to account for the completeness limit of the observed $\Sigma$ distribution.

Still, given that both saturated and IR-bright regions of IRDCs (where the extinction mapping method fails) cover very small fractional areas (Butler \& Tan 2012), we do not expect these limitations of the measured $\Sigma$ PDFs to change the overall conclusion that the simulated clouds have much higher mass fractions at high $\Sigma$. 


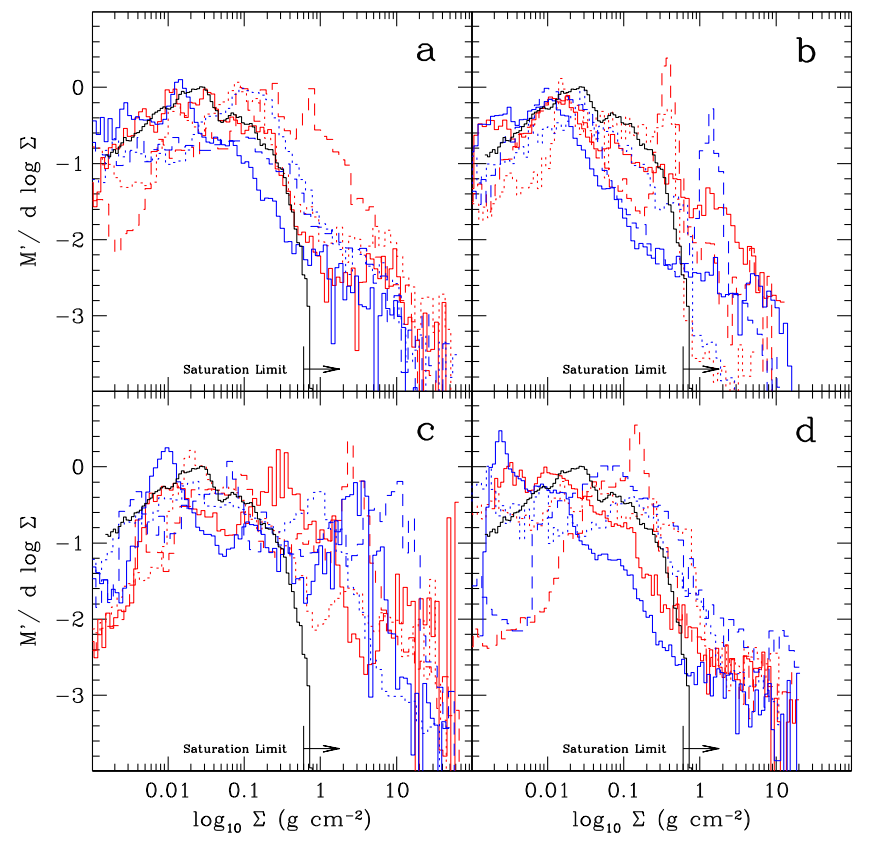

Figure 7. Mass-weighted column density PDFs for filament a (top left), filament $\mathrm{b}$ (top right), filament $\mathrm{c}$ (bottom left), and filament $\mathrm{d}$ (bottom right). Mass surface density PDFs of the inner $25 \times 25 \times 25$ pc cube (red) and $50 \times 50$ $\times 50 \mathrm{pc}$ cube (blue) calculated from the density projection along the $z$-axis are shown as solid lines, along the $y$-axis as dotted lines, and along the $x$-axis as dashed lines. Also plotted is the MIR+NIR extinction mapping PDF for Cloud C (BTK13, black solid line). The region affected by the saturation limit, i.e., where $\Sigma>\Sigma_{\text {sat }}=0.6 \mathrm{~g} \mathrm{~cm}^{-2}$, is indicated.

\subsection{Filament Structure}

We study filament properties by defining 10 individual slab regions $(10 \mathrm{pc} \times 10 \mathrm{pc} \times 5 \mathrm{pc})$ that appear as strips in projection $(10 \mathrm{pc} \times 5 \mathrm{pc})$ along each filament. These are iteratively centered on the center of mass within their $10 \mathrm{pc}$ by $10 \mathrm{pc}$ extent in the plane perpendicular to the main filament axis. Choosing a $10 \mathrm{pc}$ width for the slabs/strips is a somewhat arbitrary choice. We therefore also assess inner filament regions of $5 \mathrm{pc}$ width and $5 \mathrm{pc}$ depth (again iteratively recentered on the center of mass within their extent). These outer and inner filament regions are shown in Figure 4. We also define a "dense" filament as the material inside the inner filament with $n_{\mathrm{H}} \geqslant 10^{3} \mathrm{~cm}^{-3}$.

The mean total filament mass within the $10 \mathrm{pc}$ wide strips is $2.01 \times 10^{5} M_{\odot}$ : note that most of the $50 \mathrm{pc}$ region masses are contained within these defined filament regions.

We also define "envelope" regions that extend half the strip width on either side of the filament strips (i.e., $5 \mathrm{pc}$ for the outer filaments and $2.5 \mathrm{pc}$ for the inner filaments). They have the same depth as the filaments, i.e., 10 and $5 \mathrm{pc}$ for outer and inner cases, respectively. So with their 5 pc extent along the filament axis, this gives these total envelope region an equal volume as the filament. We define the envelope regions of the dense filament as the material inside the inner filament with $n_{\mathrm{H}}<10^{3} \mathrm{~cm}^{-3}$.

We calculate physical properties in these filament and envelope regions, which are listed in Table 2. These include volume-averaged densities, which have mean values of $n_{\mathrm{H}, f}=(3.46,4.26) \times 10^{3} \mathrm{~cm}^{-3}$ for the outer and inner filaments, respectively. Their envelope regions have mean densities $n_{\mathrm{H}, e}=(0.0414,0.449) \times 10^{3} \mathrm{~cm}^{-3}$, illustrating the decreasing, although still substantial, density contrast as we zoom into the inner filament regions.

\subsubsection{Longitudinal Structure}

The mass per unit length, $m_{l}$, profiles of the filaments are shown in Figure 8. Typical median values are $\sim 10^{3.5} M_{\odot} \mathrm{pc}^{-1}$ (about $10^{3} M_{\odot} \mathrm{pc}^{-1}$ in filament $b$ ), but with large fluctuations due to the formation of dense clumps within the filaments. Thus, we also measure the dispersion in $m_{l}$, assessed both at the finest resolution available in the simulation and on the $1 \mathrm{pc}$ scales over the entire $50 \mathrm{pc}$ length of the filaments, and report the values in Table 2 .

The values can be compared with those measured in IRDCs. Hernandez et al. (2012) measured $m_{l} \simeq 300 M_{\odot} \mathrm{pc}^{-1}$ along a $3.8 \mathrm{pc}$ length and $\sim 1 \mathrm{pc}$ wide region of IRDC $\mathrm{H}$ from the BT09 sample (G035.30-00.33) (note that this region is part of a longer filamentary structure). Dividing the filament into four strips, these showed dispersion of about $25 \%$ in their values of $\Sigma$. The Orion A filament, studied by Bally et al. (1987), extends over about $13 \mathrm{pc}$ with a similar value of $m_{l} \simeq 400 M_{\odot} \mathrm{pc}^{-1}$.

Longer filaments have been identified and studied by Jackson et al. (2010), Battersby \& Bally (2012), and Ragan et al. (2014). Jackson et al. (2010) identified an $80 \mathrm{pc}$ long filament ("Nessie") with typical values of $m_{l} \sim 500 M_{\odot} \mathrm{pc}^{-1}$, estimated assuming virial equilibrium. Battersby \& Bally (2012) found another $80 \mathrm{pc}$ long filament, identified in ${ }^{13} \mathrm{CO}$ and with mass $\sim 10^{5} M_{\odot}$ and $m_{l} \sim 10^{3} M_{\odot} \mathrm{pc}^{-1}$. Ragan et al. (2014) studied seven filaments with typical length $\sim 100 \mathrm{pc}$ and average $m_{l} \simeq 100 M_{\odot} \mathrm{pc}^{-1}$.

Comparison of these observed filament properties with those from our simulation indicates that the simulated filaments have much larger values of mass per unit length, by factors of about several to 10. A systematic and quantitative study of the dispersion in $m_{l}$ remains to be carried out for the observed filaments, but initial indications, e.g., from the Hernandez et al. (2012) study, suggest that the observed filaments have much smaller dispersions in $m_{l}$ than our simulated filaments.

\subsubsection{Filament Fragmentation}

The peaks in $m_{l}$ correspond to dense clumps that appear to have formed by fragmentation of the filament. In gravitationally bound filaments, clumps are predicted to form by fragmentation at roughly regular intervals, caused by the socalled sausage-like fluid instability (e.g., Stodolkiewicz 1963; Nagasawa 1987; Inutsuka \& Miyama 1992). In our simulated clouds, a large number of dense clumps are observed to form along the filaments at what appear to be roughly regular intervals, especially in filaments $b$ and $c$.

The ability of the simulation to properly resolve fragmentation can be assessed by reference to the Truelove et al. (1997) criterion, which requires at least 4 cells per Jeans length, $\lambda_{J}=\left(\pi c_{\mathrm{th}}^{2} /[G \rho]\right)^{1 / 2}=0.92\left(c_{\mathrm{th}} / 0.2 \mathrm{~km} \mathrm{~s}^{-1}\right)\left(n_{\mathrm{H}} / 10^{3} \mathrm{~cm}^{-3}\right)^{-1 / 2}$ pc. Thus, with our maximum resolution of $0.122 \mathrm{pc}$, we are able to resolve fragmentation down to a level when $\lambda_{J}=0.49$ pc, i.e., densities of about $n_{\mathrm{H}} \sim 3500 \mathrm{~cm}^{-3}$ for sound speeds of $0.2 \mathrm{~km} \mathrm{~s}^{-1}$. This is a relatively low density compared to the "clump" material defined in Paper I at a density threshold of $10^{5} \mathrm{~cm}^{-3}$. Note that the TT09 simulation imposed an effective temperature floor of $300 \mathrm{~K}$, corresponding to an effective sound 
Table 2

Filament Structure

\begin{tabular}{|c|c|c|c|c|c|c|c|}
\hline Filament & $\begin{array}{c}\bar{x}_{c}, \bar{y}_{c}, \bar{z}_{c} \\
(\mathrm{pc})\end{array}$ & $\begin{array}{c}M_{g} \\
\left(10^{4} M_{\odot}\right)\end{array}$ & $\begin{array}{c}m_{f} \\
\left(10^{4} M_{\odot} \mathrm{pc}^{-1}\right)\end{array}$ & $\begin{array}{c}\sigma_{m_{f}} \\
\left(10^{4} M_{\odot} \mathrm{pc}^{-1}\right)\end{array}$ & $\begin{array}{c}n_{\mathrm{H}, f} \\
\left(10^{3} \mathrm{~cm}^{-3}\right)\end{array}$ & $\begin{array}{c}n_{\mathrm{H}, e} \\
\left(10^{3} \mathrm{~cm}^{-3}\right)\end{array}$ & $\begin{array}{l}w_{\mathrm{rms}} \\
(\mathrm{pc})\end{array}$ \\
\hline $\mathrm{a}$ & $480,545,3.00$ & $17.6,16.3,15.1$ & $0.352,0.326,0.303$ & $0.337,0.338,0.339$ & $1.02,3.78,18.6$ & $0.0149,0.366,0.333$ & $1.40,0.801,0.775$ \\
\hline a1 & $458,537,1.48$ & $0.868,0.809,0.731$ & $0.174,0.162,0.146$ & $0.0264,0.0246,0.0226$ & $0.502,1.87,14.6$ & $0.00778,0.240,0.204$ & $1.67,0.820,0.803$ \\
\hline a2 & $463,530,1.81$ & $1.04,0.888,0.801$ & $0.208,0.178,0.160$ & $0.0324,0.0286,0.0278$ & $0.603,2.05,18.6$ & $0.00646,0.359,0.223$ & $1.60,0.615,0.604$ \\
\hline a3 & $468,541,2.54$ & $1.21,1.05,0.927$ & $0.242,0.209,0.185$ & $0.0760,0.0693,0.0678$ & $0.699,2.42,16.3$ & $0.00836,0.498,0.316$ & $1.51,0.673,0.604$ \\
\hline a4 & $473,541,2.55$ & $2.42,2.17,2.05$ & $0.484,0.435,0.409$ & $0.595,0.594,0.593$ & $1.40,5.03,21.9$ & $0.0147,0.671,0.373$ & $1.24,0.674,0.642$ \\
\hline a5 & $478,543,1.73$ & $1.22,1.05,0.910$ & $0.244,0.210,0.182$ & $0.0908,0.0870,0.0860$ & $0.707,2.43,10.4$ & $0.0207,0.465,0.407$ & $1.58,1.04,1.05$ \\
\hline a6 & $483,544,0.939$ & $1.21,1.16,1.02$ & $0.243,0.232,0.203$ & $0.0616,0.0604,0.0592$ & $0.705,2.69,11.4$ & $0.0189,0.158,0.423$ & $1.35,0.852,0.828$ \\
\hline a7 & $488,543,1.38$ & $6.41,6.34,6.20$ & $1.28,1.26,1.24$ & $3.25,3.25,3.24$ & $3.71,14.7,57.9$ & $0.0339,0.254,0.437$ & $0.735,0.514,0.502$ \\
\hline a8 & $493,541,1.52$ & $0.908,0.768,0.622$ & $0.182,0.154,0.124$ & $0.0767,0.0716,0.0712$ & $0.525,1.78,11.6$ & $0.0229,0.403,0.386$ & $1.65,1.11,1.08$ \\
\hline a9 & $498,541,0.938$ & $0.994,0.940,0.823$ & $0.199,0.188,0.165$ & $0.0311,0.0295,0.0269$ & $0.575,2.17,12.3$ & $0.00672,0.110,0.320$ & $1.35,0.965,0.932$ \\
\hline a10 & $503,539,1.36$ & $1.31,1.14,1.06$ & $0.263,0.229,0.213$ & $0.148,0.147,0.146$ & $0.760,2.65,11.3$ & $0.00840,0.501,0.242$ & $1.34,0.741,0.711$ \\
\hline $\mathrm{b}$ & $625,610,-3.50$ & $10.9,9.61,8.83$ & $0.219,0.192,0.176$ & $0.115,0.122,0.121$ & $0.633,2.22,1.94$ & $0.116,0.382,0.204$ & $1.78,0.885,0.786$ \\
\hline b1 & $625,588,1.78$ & $1.55,1.51,1.40$ & $0.311,0.302,0.279$ & $0.472,0.469,0.469$ & $0.899,3.49,31.4$ & $0.109,0.357,0.293$ & $1.24,0.578,0.489$ \\
\hline b2 & $627,593,1.34$ & $0.592,0.371,0.276$ & $0.118,0.0741,0.0553$ & $0.0962,0.0971,0.0966$ & $0.342,0.857,5.85$ & $0.0944,0.608,0.245$ & $2.41,1.30,1.26$ \\
\hline b3 & $628,598,0.868$ & $0.820,0.569,0.490$ & $0.164,0.114,0.0979$ & $0.237,0.239,0.239$ & $0.474,1.31,12.8$ & $0.114,0.616,0.200$ & $2.31,0.794,0.614$ \\
\hline b4 & $628,603,0.124$ & $0.594,0.457,0.374$ & $0.119,0.0913,0.0747$ & $0.197,0.193,0.193$ & $0.344,1.01,19.3$ & $0.123,0.391,0.201$ & $2.04,0.790,0.508$ \\
\hline b5 & $629,608,0.781$ & $1.16,1.11,1.05$ & $0.233,0.221,0.211$ & $0.531,0.529,0.530$ & $0.674,2.56,22.9$ & $0.124,0.179,0.139$ & $1.20,0.828,0.780$ \\
\hline b6 & $629,613,0.717$ & $1.10,1.01,0.942$ & $0.221,0.202,0.188$ & $0.266,0.266,0.267$ & $0.639,2.34,13.9$ & $0.123,0.253,0.187$ & $1.42,0.956,0.902$ \\
\hline b7 & $628,618,-1.45$ & $0.584,0.395,0.347$ & $0.117,0.0789,0.0693$ & $0.0467,0.0344,0.0349$ & $0.337,0.913,9.16$ & $0.111,0.509,0.122$ & $2.35,1.21,1.17$ \\
\hline b8 & $628,623,-0.270$ & $1.88,1.70,1.64$ & $0.376,0.341,0.327$ & $0.830,0.830,0.831$ & $1.09,3.94,32.3$ & $0.120,0.293,0.179$ & $1.53,0.667,0.610$ \\
\hline b9 & $628,628,-0.730$ & $0.549,0.457,0.373$ & $0.110,0.0914,0.0746$ & $0.151,0.150,0.151$ & $0.318,1.06,7.81$ & $0.105,0.221,0.219$ & $2.05,0.979,0.824$ \\
\hline b10 & $628,633,-0.942$ & $2.09,2.03,1.94$ & $0.418,0.406,0.387$ & $1.16,1.15,1.15$ & $1.21,4.70,39.0$ & $0.137,0.390,0.250$ & $1.24,0.741,0.695$ \\
\hline $\mathrm{c}$ & $614,0.251,20.3$ & $34.6,32.8,31.1$ & $0.692,0.656,0.621$ & $0.547,0.537,0.540$ & $2.00,7.59,50.6$ & $0.0191,0.491,0.459$ & $1.38,0.743,0.645$ \\
\hline c1 & $613,229,14.7$ & $1.05,0.926,0.803$ & $0.210,0.185,0.161$ & $0.131,0.123,0.125$ & $6.06,21.4,16.5$ & $0.0176,0.364,0.320$ & $1.80,0.953,0.755$ \\
\hline c2 & $614,234,15.7$ & $6.53,6.17,6.09$ & $1.31,1.23,1.22$ & $1.91,1.92,1.92$ & $3.77,14.3,98.2$ & $0.0243,1.06,0.208$ & $1.02,0.283,0.260$ \\
\hline c3 & $616,239,10.8$ & $5.36,5.23,5.10$ & $1.07,1.05,1.02$ & $1.30,1.30,1.30$ & $3.10,12.1,109$ & $0.0266,0.338,0.329$ & $0.712,0.235,0.199$ \\
\hline c4 & $618,244,18.9$ & $7.59,7.39,7.25$ & $1.52,1.48,1.45$ & $3.60,3.59,3.59$ & $4.39,17.1,108$ & $0.0299,0.540,0.384$ & $0.951,0.575,0.567$ \\
\hline c5 & $613,249,21.1$ & $1.09,0.952,0.692$ & $0.217,0.190,0.138$ & $0.124,0.126,0.113$ & $0.629,2.20,12.9$ & $0.0162,0.348,0.689$ & $2.01,1.11,0.913$ \\
\hline c6 & $612,254,20.8$ & $6.13,5.86,5.61$ & $1.23,1.17,1.12$ & $2.95,2.95,2.94$ & $3.54,13.6,53.8$ & $0.0193,0.734,0.750$ & $1.03,0.676,0.660$ \\
\hline c7 & $615,259,20.6$ & $1.02,0.848,0.662$ & $0.204,0.170,0.132$ & $0.131,0.129,0.129$ & $0.590,1.96,20.4$ & $0.00959,0.535,0.467$ & $1.56,0.798,0.668$ \\
\hline c8 & $615,264,24.1$ & $3.80,3.73,3.52$ & $0.761,0.747,0.704$ & $1.80,1.79,1.79$ & $2.20,8.64,53.9$ & $0.00759,0.245,0.579$ & $0.903,0.697,0.678$ \\
\hline c9 & $614,269,24.1$ & $0.741,0.471,0.320$ & $0.148,0.0943,0.0640$ & $0.110,0.106,0.103$ & $0.428,1.09,15.8$ & $0.0328,0.589,0.369$ & $2.62,1.25,0.955$ \\
\hline $\mathrm{c} 10$ & $613,274,27.7$ & $1.26,1.21,1.02$ & $0.252,0.242,0.205$ & $0.291,0.291,0.291$ & $0.729,2.79,17.3$ & $0.00758,0.157,0.492$ & $1.20,0.849,0.800$ \\
\hline $\mathrm{d}$ & $785,200,10.0$ & $17.1,15.0,13.2$ & $0.342,0.300,0.264$ & $0.188,0.185,0.184$ & $0.990,3.46,15.9$ & $0.0155,0.557,0.520$ & $1.55,0.808,0.772$ \\
\hline d1 & $782,178,5.98$ & $1.20,0.946,0.816$ & $0.239,0.189,0.163$ & $0.0530,0.0492,0.0484$ & $0.692,2.19,11.3$ & $0.0290,0.631,0.363$ & $1.78,0.759,0.713$ \\
\hline d2 & $783,183,6.05$ & $1.28,1.04,0.919$ & $0.257,0.210,0.184$ & $0.0780,0.0644,0.0648$ & $0.742,2.40,7.80$ & $0.0148,0.630,0.382$ & $1.66,0.886,0.854$ \\
\hline d3 & $784,188,6.08$ & $1.63,1.52,1.35$ & $0.326,0.305,0.270$ & $0.0944,0.0924,0.0906$ & $0.943,3.52,10.7$ & $0.0198,0.331,0.574$ & $1.31,0.814,0.797$ \\
\hline d4 & $785,193,6.12$ & $1.11,1.01,0.826$ & $0.222,0.202,0.165$ & $0.0445,0.0417,0.0376$ & $0.642,2.34,8.84$ & $0.0122,0.282,0.548$ & $1.42,0.872,0.828$ \\
\hline d5 & $786,198,6.33$ & $2.36,2.26,2.06$ & $0.471,0.453,0.413$ & $0.742,0.742,0.741$ & $1.36,5.24,29.0$ & $0.0135,0.311,0.555$ & $1.01,0.489,0.455$ \\
\hline d6 & $786,203,6.39$ & $0.912,0.753,0.547$ & $0.182,0.151,0.109$ & $0.0278,0.0219,0.0167$ & $0.528,1.74,12.7$ & $0.00820,0.501,0.531$ & $1.63,0.788,0.687$ \\
\hline d7 & $786,208,6.84$ & $3.06,2.86,2.64$ & $0.612,0.572,0.528$ & $1.24,1.24,1.24$ & $1.77,6.61,33.9$ & $0.00972,0.532,0.616$ & $1.17,0.576,0.558$ \\
\hline
\end{tabular}


Table 2

(Continued)

\begin{tabular}{|c|c|c|c|c|c|c|c|}
\hline Filament & $\begin{array}{c}\bar{x}_{c}, \bar{y}_{c}, \bar{z}_{c} \\
\quad(\mathrm{pc})\end{array}$ & $\begin{array}{c}M_{g} \\
\left(10^{4} M_{\odot}\right)\end{array}$ & $\begin{array}{c}m_{f} \\
\left(10^{4} M_{\odot} \mathrm{pc}^{-1}\right)\end{array}$ & $\begin{array}{c}\sigma_{m_{f}} \\
\left(10^{4} M_{\odot} \mathrm{pc}^{-1}\right)\end{array}$ & $\begin{array}{c}n_{\mathrm{H}, f} \\
\left(10^{3} \mathrm{~cm}^{-3}\right)\end{array}$ & $\begin{array}{c}n_{\mathrm{H}, e} \\
\left(10^{3} \mathrm{~cm}^{-3}\right)\end{array}$ & $\begin{array}{l}w_{\text {rms }} \\
(\mathrm{pc})\end{array}$ \\
\hline $\mathrm{d} 8$ & $785,213,7.28$ & $0.960,0.790,0.599$ & $0.192,0.158,0.120$ & $0.0345,0.0295,0.0263$ & $0.555,1.83,8.38$ & $0.0134,0.424,0.531$ & $1.76,0.913,0.877$ \\
\hline d9 & $784,218,7.68$ & $3.53,3.16,2.99$ & $0.706,0.632,0.598$ & $1.34,1.35,1.35$ & $2.04,7.31,32.0$ & $0.00491,0.933,0.506$ & $1.31,0.665,0.654$ \\
\hline $\mathrm{d} 10$ & $782,223,8.97$ & $1.08,0.652,0.454$ & $0.216,0.130,0.0907$ & $0.0740,0.0297,0.0281$ & $0.625,1.51,4.63$ & $0.0292,0.992,0.593$ & $2.44,1.32,1.30$ \\
\hline
\end{tabular}

Note.

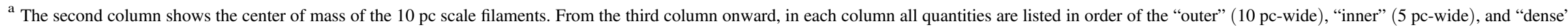

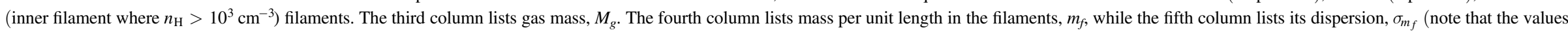

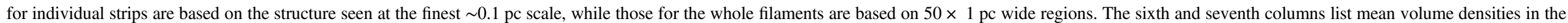
filament and envelope regions. The eighth column lists rms lateral widths. 
Table 3

Filament Kinematics $^{\mathrm{a}}$

\begin{tabular}{|c|c|c|c|c|c|c|c|c|c|c|c|}
\hline $\begin{array}{l}\begin{array}{l}\text { Filam- } \\
\text { ent }\end{array} \\
\end{array}$ & $\begin{array}{c}\bar{v}_{\mathrm{x}}^{\mathrm{b}} \\
\left(\mathrm{km} \mathrm{s}^{-1}\right)\end{array}$ & $\begin{array}{c}\overline{v y}_{\mathrm{y}}^{\mathrm{b}} \\
\left(\mathrm{km} \mathrm{s}^{-1}\right)\end{array}$ & $\begin{array}{c}\bar{v}_{\mathrm{z}}^{\mathrm{b}} \\
\left(\mathrm{km} \mathrm{s}^{-1}\right)\end{array}$ & $\begin{array}{c}\bar{\sigma}_{f, x} \\
\left(\mathrm{~km} \mathrm{~s}^{-1}\right)\end{array}$ & $\begin{array}{c}\bar{\sigma}_{f, y} \\
\left(\mathrm{~km} \mathrm{~s}^{-1}\right)\end{array}$ & $\begin{array}{c}\bar{\sigma}_{f, z} \\
\left(\mathrm{~km} \mathrm{~s}^{-1}\right)\end{array}$ & $\begin{array}{c}\bar{\sigma}_{e, x} \\
\left(\mathrm{~km} \mathrm{~s}^{-1}\right)\end{array}$ & $\begin{array}{c}\bar{\sigma}_{e, y} \\
\left(\mathrm{~km} \mathrm{~s}^{-1}\right)\end{array}$ & $\begin{array}{c}\bar{\sigma}_{e, z} \\
\left(\mathrm{~km} \mathrm{~s}^{-1}\right)\end{array}$ & $\mathrm{m}_{\mathrm{f}} / \overline{\mathrm{m}}_{\mathrm{vir}}$ & $\log _{10} P_{e} / P_{f}$ \\
\hline a & $-1.3,-1.5,-0.97$ & $-0.22,0.24,0.53$ & $0.42,0.43,0.44$ & $4.16,4.09,3.47$ & $4.75,4.61,4.24$ & $2.79,2.78,2.68$ & $2.20,2.49,2.79$ & $5.23,2.83,5.02$ & $2.51,1.54,2.43$ & $0.27,0.90,0.24$ & $-1.7,-1.4,-1.4$ \\
\hline al & $-8.0,-8.1,-7.8$ & $2.9,2.9,3.1$ & $0.28,0.27,0.21$ & $3.37,3.19,2.95$ & $2.42,2.46,2.30$ & $0.855,0.652,0.502$ & $2.11,1.21,2.16$ & $4.16,1.50,3.73$ & $2.28,1.25,1.30$ & $0.64,0.57,0.60$ & $-1.3,-1.3,-1.6$ \\
\hline $\mathrm{a}_{2}$ & $-6.7,-7.4,-7.0$ & $3.6,4.0,4.2$ & $0.19,0.22,0.16$ & $4.16,4.05,3.83$ & $2.56,2.47,2.33$ & $0.957,0.814,0.714$ & $2.80,1.27,2.41$ & $5.53,1.49,3.83$ & $2.92,1.19,1.29$ & $0.68,0.63,0.63$ & $-1.3,-1.2,-1.9$ \\
\hline a3 & $-3.2,-3.3,-2.7$ & $2.5,3.0,3.5$ & $-0.022,-0.081,-0.12$ & $4.22,4.26,3.81$ & $4.13,4.22,3.92$ & $1.63,1.63,1.63$ & $2.74,1.10,1.60$ & $5.08,2.22,4.75$ & $2.83,1.02,1.59$ & $0.30,0.25,0.26$ & $-1.7,-1.2,-1.7$ \\
\hline a4 & $-3.9,-4.3,-4.1$ & $3.9,4.6,5.0$ & $-0.53,-0.58,-0.67$ & $4.18,4.15,4.01$ & $5.53,5.31,5.15$ & $4.25,4.46,4.53$ & $2.82,2.31,2.05$ & $4.61,3.12,4.96$ & $3.02,1.24,2.70$ & $0.34,0.33,0.33$ & $-2.1,-1.3,-1.8$ \\
\hline a5 & $-3.3,-3.8,-3.2$ & $0.47,1.5,2.0$ & $0.49,0.59,0.56$ & $4.64,4.73,4.38$ & $4.65,3.84,3.49$ & $2.15,2.25,2.21$ & $2.60,1.97,2.08$ & $6.02,2.96,5.12$ & $2.93,1.29,2.47$ & $0.24,0.31,0.32$ & $-1.3,-0.94,-1.2$ \\
\hline a6 & $1.9,2.2,3.2$ & $-1.5,-1.0,-0.37$ & $0.43,0.35,0.37$ & $4.05,3.85,2.25$ & $4.80,4.47,3.65$ & $1.95,1.86,1.74$ & $2.49,2.28,2.28$ & $4.98,3.71,5.62$ & $2.37,1.82,2.51$ & $0.23,0.25,0.33$ & $-1.5,-1.4,-0.94$ \\
\hline a7 & $1.5,1.5,1.7$ & $1.9,2.0,2.3$ & $-0.31,-0.34,-0.35$ & $5.36,5.35,5.28$ & $10.2,10.2,10.1$ & $9.79,9.84,9.93$ & $2.21,3.21,2.93$ & $6.08,3.98,5.37$ & $2.92,2.31,3.63$ & $0.26,0.26,0.26$ & $-2.5,-2.6,-2.3$ \\
\hline a8 & $-2.2,-2.8,-2.0$ & $-4.6,-3.7,-3.8$ & $1.6,1.7,2.1$ & $4.56,4.39,3.92$ & $5.27,5.20,4.67$ & $2.48,2.51,2.15$ & $1.77,4.18,4.21$ & $5.59,3.01,4.77$ & $1.94,1.69,3.19$ & $0.14,0.12,0.12$ & $-1.3,-1.2,-1.1$ \\
\hline a9 & $2.9,3.0,3.8$ & $-5.9,-5.8,-5.7$ & $0.92,0.88,0.92$ & $3.54,3.41,2.21$ & $3.55,3.52,2.81$ & $1.65,1.50,1.22$ & $1.31,4.96,4.34$ & $5.77,3.45,5.08$ & $2.04,2.24,2.74$ & $0.34,0.33,0.45$ & $-1.5,-1.3,-0.84$ \\
\hline a10 & $7.5,7.8,8.4$ & $-5.6,-5.1,-4.9$ & $1.2,1.3,1.2$ & $3.56,3.55,2.06$ & $4.38,4.45,3.96$ & $2.19,2.25,2.19$ & $1.19,2.40,3.88$ & $4.49,2.87,6.97$ & $1.88,1.38,2.90$ & $0.30,0.25,0.29$ & $-1.9,-1.1,-1.1$ \\
\hline b & $-0.015,-0.24,-0.23$ & $-0.64,-0.78,-0.86$ & $0.42,0.23,0.17$ & $3.37,3.31,3.31$ & $1.87,1.94,1.91$ & $3.25,2.46,3.52$ & $3.41,2.13,2.88$ & $1.10,1.09,1.83$ & $1.15,1.28,2.64$ & $0.56,1.1,0.49$ & $-0.67,-1.1,-2.0$ \\
\hline b1 & $-2.4,-2.3,-2.3$ & $-6.6,-6.7,-6.7$ & $0.24,0.27,0.24$ & $3.56,3.57,3.64$ & $2.06,2.08,2.09$ & $3.48,3.60,3.63$ & $3.97,1.57,2.55$ & $1.69,1.45,1.78$ & $1.10,1.20,3.18$ & $0.53,0.51,0.45$ & $-0.82,-1.7,-2.3$ \\
\hline b2 & $-2.2,-2.4,-2.4$ & $-6.4,-7.4,-8.0$ & $0.80,0.14,0.038$ & $2.82,2.46,2.63$ & $2.65,2.84,2.88$ & $3.12,3.65,3.85$ & $3.53,1.16,1.84$ & $1.18,0.936,1.98$ & $1.26,1.18,2.98$ & $0.32,0.26,0.17$ & $-0.36,-0.80,-1.7$ \\
\hline b3 & $-0.64,-1.2,-1.1$ & $-3.9,-4.1,-4.1$ & $0.72,0.27,0.24$ & $2.69,2.46,2.56$ & $1.38,1.57,1.61$ & $2.56,2.87,2.93$ & $3.20,1.08,1.70$ & $0.941,0.656,1.24$ & $0.908,1.17,2.52$ & $0.49,0.40,0.32$ & $-0.47,-1.1,-2.2$ \\
\hline b4 & $-0.39,-0.75,-0.66$ & $-3.2,-3.4,-3.7$ & $0.27,-0.065,-0.26$ & $2.42,2.47,2.39$ & $1.46,1.56,1.45$ & $2.23,2.40,2.40$ & $4.30,1.96,2.77$ & $1.21,0.972,1.52$ & $1.24,1.04,2.15$ & $0.44,0.32,0.28$ & $0.051,-0.63,-1.9$ \\
\hline b5 & $-0.15,0.10,-0.13$ & $0.82,0.87,0.90$ & $0.21,0.17,0.11$ & $3.89,3.98,3.98$ & $1.86,1.88,1.87$ & $3.90,3.99,4.04$ & $3.81,2.80,3.87$ & $1.07,1.01,1.94$ & $1.30,1.14,2.59$ & $0.33,0.30,0.29$ & $-0.75,-1.5,-2.2$ \\
\hline b6 & $0.61,0.60,0.62$ & $-0.018,-0.063,-0.067$ & $0.33,0.28,0.25$ & $2.52,2.47,2.43$ & $1.70,1.76,1.78$ & $2.47,2.55,2.53$ & $3.72,1.97,3.02$ & $0.890,0.727,1.48$ & $1.23,1.10,2.77$ & $0.75,0.71,0.69$ & $-0.38,-1.2,-1.7$ \\
\hline b7 & $1.7,1.3,1.4$ & $2.9,3.1,3.4$ & $0.80,0.77,0.72$ & $2.81,2.82,2.62$ & $1.73,1.88,1.80$ & $2.07,2.35,2.42$ & $4.33,2.20,3.88$ & $0.996,1.38,1.56$ & $0.958,1.45,1.79$ & $0.32,0.21,0.22$ & $-0.11,-0.47,-1.5$ \\
\hline b8 & $0.77,0.40,0.41$ & $4.2,4.3,4.4$ & $-0.12,-0.24,-0.28$ & $4.59,4.66,4.71$ & $1.85,1.85,1.85$ & $4.23,4.41,4.45$ & $2.67,2.61,3.17$ & $0.959,1.04,1.91$ & $1.26,1.44,3.07$ & $0.38,0.34,0.32$ & $-1.4,-1.6,-2.6$ \\
\hline b9 & $1.5,0.69,0.73$ & $1.7,1.3,1.1$ & $0.78,0.55,0.53$ & $2.81,2.61,2.50$ & $1.70,1.60,1.53$ & $2.50,2.68,2.73$ & $2.49,3.61,3.06$ & $0.971,0.974,1.58$ & $1.00,1.40,2.45$ & $0.30,0.29,0.26$ & $-0.58,-0.40,-1.4$ \\
\hline b10 & $1.1,1.1,1.1$ & $4.2,4.2,4.3$ & $0.17,0.13,0.12$ & $5.55,5.58,5.68$ & $2.32,2.34,2.26$ & $5.97,6.06,6.17$ & $2.03,2.38,2.93$ & $1.08,1.76,3.29$ & $1.22,1.69,2.86$ & $0.29,0.28,0.26$ & $-1.8,-1.8,-2.8$ \\
\hline $\mathrm{c}$ & $-1.2,-0.58,-2.9$ & $-0.14,-0.074,-1.6$ & $-0.64,-0.72,0.31$ & 7.14,6.99,6.95 & $5.28,5.31,5.21$ & $4.96,5.04,5.02$ & $2.91,3.39,4.75$ & $2.27,2.82,4.76$ & $1.82,1.74,3.45$ & $1.8,1.3,0.57$ & $-2.7,-1.7,-2.2$ \\
\hline $\mathrm{cl}$ & 4.6, 5.4, 2.9 & $-1.9,-1.3,1.3$ & $1.6,1.6,1.6$ & $5.54,5.24,4.96$ & $4.31,4.24,4.32$ & $1.92,1.97,1.84$ & $2.84,3.13,4.09$ & $2.74,2.24,3.71$ & $1.44,1.43,2.67$ & $0.15,0.15,0.14$ & $-2.1,-1.2,-1.9$ \\
\hline $\mathrm{c} 2$ & $1.2,1.6,-0.95$ & $2.6,3.1,-0.71$ & $0.96,0.94,3.2$ & $9.77,9.90,9.93$ & $10.7,10.7,10.8$ & $3.15,3.21,3.17$ & $3.91,3.98,4.29$ & $3.19,2.87,6.10$ & $2.58,1.84,4.21$ & $0.29,0.27,0.27$ & $-3.0,-1.9,-3.4$ \\
\hline c3 & $3.2,3.4,1.3$ & $-0.29,-1.5,-3.5$ & $-0.060,-0.13,0.43$ & $8.35,8.36,8.38$ & $8.57,8.62,8.66$ & $2.85,2.84,2.80$ & $3.18,2.68,5.06$ & $3.16,2.93,5.07$ & $1.73,1.31,3.88$ & $0.33,0.32,0.31$ & $-2.9,-2.5,-3.0$ \\
\hline c4 & $1.5,1.6,-1.3$ & $0.12,0.15,-1.8$ & $-0.068,-0.14,0.14$ & $10.1,10.2,10.3$ & $3.91,3.81,3.75$ & $9.30,9.41,9.48$ & $2.93,4.94,6.45$ & $2.73,4.38,5.91$ & $1.41,1.37,3.86$ & $0.32,0.30,0.29$ & $-3.2,-2.1,-2.9$ \\
\hline c5 & $-4.2,-4.2,-5.0$ & $-3.3,-3.2,-4.0$ & $-1.3,-1.8,-0.72$ & $5.01,4.91,4.57$ & $4.28,4.32,4.28$ & $4.10,3.98,4.06$ & $3.80,4.31,5.06$ & $2.76,3.13,4.44$ & $2.49,1.95,3.73$ & $0.19,0.17,0.14$ & $-1.8,-0.92,-1.2$ \\
\hline c6 & $1.3,1.5,-1.5$ & $-2.4,-2.3,-4.6$ & $-0.71,-0.77,1.1$ & $8.85,8.92,9.03$ & $4.42,4.43,4.43$ & $8.42,8.60,8.75$ & $2.33,3.64,5.20$ & $1.28,2.98,3.87$ & $1.50,1.87,3.78$ & $0.34,0.32,0.30$ & $-3.4,-2.1,-2.3$ \\
\hline c7 & $-4.0,-3.0,-5.0$ & $-2.5,-2.6,-4.1$ & $0.51,0.62,1.5$ & $4.01,3.61,3.69$ & $2.72,2.80,2.57$ & $2.06,2.18,2.15$ & $2.32,3.16,3.23$ & $1.85,2.94,3.33$ & $1.62,1.25,2.24$ & $0.27,0.28,0.21$ & $-2.3,-0.68,-1.8$ \\
\hline c8 & $-4.2,-4.2,-7.8$ & $3.5,3.4,0.15$ & $0.16,0.22,-0.25$ & $7.21,7.25,7.37$ & $3.00,3.01,2.87$ & $6.64,6.68,6.84$ & $2.51,2.36,3.93$ & $2.37,1.82,4.33$ & $2.06,1.70,2.98$ & $0.314,0.305,0.279$ & $-3.4,-2.5,-2.5$ \\
\hline c9 & $-5.5,-2.2,-4.0$ & $2.3,2.8,0.53$ & $0.97,1.1,2.5$ & $6.12,5.02,4.81$ & $3.59,4.04,3.99$ & $2.50,2.87,3.01$ & $2.59,3.00,4.70$ & $1.08,2.03,3.98$ & $1.38,2.05,2.54$ & $0.085,0.081,0.060$ & $-1.9,-0.72,-1.7$ \\
\hline c10 & $-5.5,-5.6,-8.1$ & $0.41,0.72,0.85$ & $-8.4,-8.8,-6.4$ & $6.39,6.51,6.49$ & $7.31,7.12,6.47$ & $8.68,8.62,8.07$ & $2.72,2.66,5.52$ & $1.50,2.88,6.83$ & $1.94,2.60,4.57$ & $0.13,0.12,0.11$ & $-2.7,-2.0,-1.7$ \\
\hline d & $1.5,1.4,2.5$ & $0.88,0.81,0.16$ & $0.24,-0.32,0.42$ & $5.63,5.49,3.73$ & $5.01,4.96,3.26$ & $3.11,3.23,3.24$ & $2.49,4.25,6.81$ & $3.21,3.93,6.59$ & $1.28,5.01,1.78$ & $1.7,0.39,0.13$ & $-2.6,-0.99,-0.84$ \\
\hline d1 & $3.8,4.0,6.2$ & $0.61,0.99,0.61$ & $0.73,1.0,1.4$ & $5.61,5.40,3.29$ & $3.56,3.40,1.85$ & $1.76,1.78,1.71$ & $1.93,4.15,7.63$ & $3.02,2.73,5.01$ & $1.21,1.03,1.96$ & $0.16,0.14,0.32$ & $-2.3,-0.77,-0.76$ \\
\hline d2 & $4.0,4.1,5.5$ & $0.63,0.90,1.3$ & $0.55,0.76,0.64$ & $5.04,4.83,2.48$ & $3.41,3.38,1.79$ & $1.89,1.97,1.90$ & $1.55,4.08,8.32$ & $2.79,2.85,6.10$ & $1.18,4.83,1.87$ & $0.22,0.19,0.64$ & $-2.7,-0.73,-0.26$ \\
\hline d3 & $3.5,3.7,4.7$ & $1.6,1.8,1.8$ & $0.37,0.38,0.41$ & $4.59,4.30,2.20$ & $3.93,3.71,2.20$ & $2.22,2.27,2.26$ & $1.74,4.39,6.84$ & $2.81,3.20,5.52$ & $1.17,4.30,1.91$ & $0.33,0.35,1.2$ & $-2.5,-1.0,-0.29$ \\
\hline $\mathrm{d} 4$ & $2.6,3.0,4.2$ & $0.77,1.2,2.0$ & $0.30,0.32,0.24$ & $5.43,4.96,2.24$ & $4.53,4.13,2.25$ & $1.87,1.89,1.69$ & $1.71,4.75,6.47$ & $2.74,3.80,6.14$ & $1.23,4.96,1.50$ & $0.16,0.18,0.71$ & $-2.7,-0.96,-0.29$ \\
\hline d5 & $2.9,3.0,3.5$ & $2.9,3.1,2.0$ & $0.35,0.35,-0.051$ & $5.67,5.55,4.52$ & $5.13,4.94,3.74$ & $4.28,4.36,4.48$ & $1.60,4.62,6.20$ & $2.57,3.96,6.09$ & $1.10,5.55,1.73$ & $0.32,0.32,0.43$ & $-3.1,-1.4,-1.4$ \\
\hline
\end{tabular}


Table 3

(Continued)

\begin{tabular}{|c|c|c|c|c|c|c|c|c|c|c|c|}
\hline $\begin{array}{l}\text { Filam- } \\
\text { ent }\end{array}$ & $\begin{array}{c}\bar{v}_{\mathrm{x}}^{\mathrm{b}} \\
\left(\mathrm{km} \mathrm{s}^{-1}\right)\end{array}$ & $\begin{array}{c}\bar{\nu}_{\mathrm{y}}^{\mathrm{b}} \\
\left(\mathrm{km} \mathrm{s}^{-1}\right)\end{array}$ & $\begin{array}{c}\bar{v}_{\mathrm{z}}^{\mathrm{b}} \\
\left(\mathrm{km} \mathrm{s}^{-1}\right)\end{array}$ & $\begin{array}{c}\bar{\sigma}_{f, x} \\
\left(\mathrm{~km} \mathrm{~s}^{-1}\right)\end{array}$ & $\begin{array}{c}\bar{\sigma}_{f, y} \\
\left(\mathrm{~km} \mathrm{~s}^{-1}\right)\end{array}$ & $\begin{array}{r}\bar{\sigma}_{f, z} \\
\left(\mathrm{~km} \mathrm{~s}^{-1}\right) \\
\end{array}$ & $\begin{array}{c}\bar{\sigma}_{e, x} \\
\left(\mathrm{~km} \mathrm{~s}^{-1}\right)\end{array}$ & $\begin{array}{c}\bar{\sigma}_{e, y} \\
\left(\mathrm{~km} \mathrm{~s}^{-1}\right) \\
\end{array}$ & $\begin{array}{c}\bar{\sigma}_{e, z} \\
\left(\mathrm{~km} \mathrm{~s}^{-1}\right)\end{array}$ & $\mathrm{m}_{\mathrm{f}} / \overline{\mathrm{m}}_{\mathrm{vir}}$ & $\log _{10} P_{e} / P_{f}$ \\
\hline d6 & $0.11,-0.19,2.4$ & $0.016,0.065,1.9$ & $0.029,0.043,0.25$ & $6.23,6.18,2.86$ & $6.37,6.47,3.30$ & $1.88,1.95,1.68$ & $1.81,4.49,6.09$ & $2.18,4.69,7.02$ & $1.07,6.18,1.64$ & $0.10,0.085,0.29$ & $-2.9,-0.82,-0.72$ \\
\hline d7 & $1.4,1.5,2.4$ & $2.5,2.6,-0.56$ & $0.088,0.12,0.18$ & $6.63,6.71,5.98$ & $5.77,5.75,4.31$ & $5.80,5.98,6.18$ & $4.61,3.98,6.58$ & $3.17,4.62,6.54$ & $1.41,6.71,1.76$ & $0.30,0.27,0.32$ & $-2.6,-1.6,-1.7$ \\
\hline d8 & $-2.4,-2.8,-0.26$ & $-1.1,-1.6,0.51$ & $-0.067,-0.0039,0.47$ & $5.77,5.85,4.22$ & $7.05,7.33,4.88$ & $2.09,2.17,1.99$ & $5.77,4.21,6.31$ & $4.46,3.77,8.37$ & $1.90,5.85,1.81$ & $0.12,0.099,0.14$ & $-1.6,-0.92,-0.85$ \\
\hline d9 & $-0.31,-0.24,-1.2$ & $1.4,1.4,-3.8$ & $0.083,0.14,0.52$ & $6.55,6.76,6.45$ & $5.90,5.98,5.07$ & $6.10,6.42,6.58$ & $3.42,3.86,7.02$ & $4.74,4.87,7.60$ & $1.42,6.76,1.87$ & $0.35,0.30,0.31$ & $-3.2,-1.4,-1.7$ \\
\hline d10 & $-4.7,-6.3,-5.3$ & $-2.5,-4.8,-3.7$ & $-0.35,-6.0,0.22$ & $4.94,4.74,3.56$ & $6.53,6.89,4.83$ & $2.19,2.45,2.66$ & $4.07,3.94,6.11$ & $4.86,4.66,9.33$ & $1.69,4.74,1.81$ & $0.19,0.13,0.15$ & $-1.5,-0.34,-0.42$ \\
\hline
\end{tabular}

w Notes.

${ }^{\text {a }}$ From the second column onward, in each column all quantities are linked in order of the "outer" (10 pc wide), "inner" $\left(5\right.$ pc wide), and "dense" (inner filament where $\left.n_{\mathrm{H}}>10 \mathrm{~cm}^{-3}\right)$ filaments.

${ }^{\mathrm{b}}$ Mass-weighted mean velocities. 
speed of $1.8 \mathrm{~km} \mathrm{~s}^{-1}$, to mimic effects of microturbulence. The actual fragmentation of the GMCs and filaments involves gas that is quickly cooling from a few hundred $\mathrm{K}$ down toward $10 \mathrm{~K}$, but with the structures also buffeted by turbulent motions (with speeds from 1 to $10 \mathrm{~km} \mathrm{~s}^{-1}$ ) imparted from the cloud bulk motions and their gravitational collapse. Given such conditions, we cannot be confident that the actual fragmentation we see in the simulation is free of numerical artifacts, and so the following properties of the spacing of the clumps should be treated with caution. Comparing the $0.5 \mathrm{pc}$ resolution simulations with the $0.1 \mathrm{pc}$ resolution ones, we do identify most of the same clumps forming in the same locations, with the fragmentation of only a few of the smaller clumps unresolved. However, given the above considerations, we cannot be fully confident that the actual fragmentation we see in the simulation is free of numerical artifacts, and so the following properties of the spacing of the clumps should be treated with caution.

To investigate clump spacing quantitatively, we identify clumps using the clump-finding routine of Smith et al. (2008) that is within the $y t$ software package (Turk et al. 2011). This routine identifies topologically connected structures using a recursive contouring algorithm, given a density range and density increment. One can also specify a minimum number of contiguous cells to be considered a clump. We set this threshold to 50 cells. We choose a density increment of one order of magnitude in $n_{\mathrm{H}}$, ranging from $n_{\mathrm{H}}=10^{5} \mathrm{~cm}^{-3}$ to $n_{\mathrm{H}}=10^{8} \mathrm{~cm}^{-3}$, near the maximum density reached by our simulation. The routine identifies $7,11,11$, and 5 clumps in filaments $\mathrm{a}-\mathrm{d}$, respectively. The 3D distance between each clump center and the nearest clump along the filament is calculated. The mean separations in each filament are 5.30, $5.26,5.87$, and $7.84 \mathrm{pc}$ with dispersions of $3.42,3.27,4.29$, and $4.56 \mathrm{pc}$ for filaments $\mathrm{a}-\mathrm{d}$, respectively. We find a mean separation for all clumps of $5.54 \mathrm{pc}$, with dispersion of $3.60 \mathrm{pc}$.

Note that the number and properties of the clumps are sensitive to the choice of threshold density and the required minimum number of cells. Considering filament $\mathrm{c}$, if the density threshold is raised and lowered by a factor of two, then the number of clumps changes from 11 to 9 and 15 , respectively. If the minimum required number of cells is raised/lowered by a factor of two, then the number of clumps changes from 11 to 9 and 15, respectively.

Fragmentation at regular intervals has been seen in large filamentary IRDCs. For example, Jackson et al. (2010) find a fairly regular spacing of $\sim 4.5 \mathrm{pc}$ between individual dense clumps. While this qualitative agreement is noteworthy, given the numerical difficulties of properly resolving fragmentation and the sensitivity of results to clump identification method parameters, we cannot draw any firm conclusion from this result. Still, the fragmentation spacing we observe in the simulation serves as a point of comparison for future numerical studies that achieve higher resolution and that include magnetic fields.

\subsubsection{Lateral Structure}

The width of filaments in the ISM can provide insight into the conditions from which they formed. Arzoumanian et al. (2011) found a characteristic width of $\sim 0.1 \mathrm{pc}$ for a sample of 27 filaments in the Herschel Gould Belt Survey toward the IC 5146 molecular cloud, suggesting that the dissipation of largescale turbulence to leave thermally dominated structures may have played an important role in the filaments' formation (however, see Smith et al. 2014). It remains to be established if such a common scale of filament width is relevant to more massive IRDCs, although inspection of the BT12 maps of 10 IRDCs suggests that there are a range of widths, extending to larger values.

Our simulated filaments, with minimum resolution of about $0.1 \mathrm{pc}$, are not well resolved enough to measure scales of filament width down to the level claimed by Arzoumanian et al. (2011). For each of the $10 \mathrm{pc}$ wide and deep strips covering our simulated filaments, the mean $\Sigma$ profiles are calculated perpendicular to the filament axis and displayed in Figure 9. As can be seen from this figure and also from Figure 4, a variety of profiles are present. The peak $\Sigma$ values range from $\sim 0.1$ to greater than $1 \mathrm{~g} \mathrm{~cm}^{-2}$, averaged over the $5 \mathrm{pc}$ wide extent of the strip regions. Multiple peaks can be present, sometimes due to multiple diffuse sub-filaments (e.g., $a 1, a 2$, a3) or multiple clumps (e.g., a4). The peaks are often dominated by the presence of a single clump, and the overall profile can be affected by how clumps happen to be distributed in these strip regions (e.g., $c 2$ ).

In Figure 9 we also show the lateral $\Sigma$ profiles of strips from IRDC filaments $\mathrm{F}$ and $\mathrm{H}$ that were defined by Hernandez \& Tan (2011), but using the latest combined MIR+NIR extinction maps of Kainulainen \& Tan (2013). One notices that the peak $\Sigma$ values are smaller in the observed filaments, but a range of widths are present that is similar to some of the peaks shown by the simulated filaments. The four strips of IRDC $\mathrm{H}$ show relatively similar lateral profiles, while IRDC $\mathrm{F}$ shows a wider variety, more similar to the simulated filaments.

For a more quantitative comparison we evaluate the massweighted "rms lateral width," $w_{\text {rms }}$, i.e., the rms displacement of filament strip material from its center of mass in the lateral direction. On scales of the $5 \mathrm{pc}$ wide strips extending $10 \mathrm{pc}$ laterally, the simulated filaments have average ( \pm dispersion) rms lateral widths of $1.40 \pm 0.264 \mathrm{pc}, 1.78 \pm 0.475 \mathrm{pc}$, $1.38 \pm 0.572 \mathrm{pc}$, and $1.55 \pm 0.385 \mathrm{pc}$ for $a-d$, respectively. On scales of $1 \mathrm{pc}$-wide strips the rms lateral widths are $1.06 \pm 0.302 \mathrm{pc}, \quad 1.46 \pm 0.718 \mathrm{pc}, \quad 0.951 \pm 0.575 \mathrm{pc}, \quad$ and $1.39 \pm 0.451 \mathrm{pc}$. Note that this is the rms width of material within $\pm 5 \mathrm{pc}$ of the center of mass of each strip.

We also calculate this width for the IRDCs F and $\mathrm{H}$ using the strips defined in Hernandez et al. (2011), which have widths of $\sim 7.5 \mathrm{pc}$ and $\sim 3.1 \mathrm{pc}$, respectively. For IRDC F, we find rms lateral widths of $0.719 \pm 0.517 \mathrm{pc}$, and for IRDC $\mathrm{H}$ we find $0.409 \pm 0.317 \mathrm{pc}$. We also consider the $\pm 5 \mathrm{pc}$ scale, finding similar widths of $0.657 \pm 0.482 \mathrm{pc}$ and $0.406 \pm 0.290 \mathrm{pc}$ for IRDCs $\mathrm{F}$ and $\mathrm{H}$, respectively. These are factors of a few smaller than the simulated filament at the $1 \mathrm{pc}$-wide strip scale. These results, including for indivdiual strips and for inner and dense filaments, are also listed in Table 2.

\subsection{Filament and Clump Kinematics}

We show the position-velocity $(p-v)$ diagrams for filaments $a-d$ in Figure 10, with the position coordinate ranging along the $50 \mathrm{pc}$ length of the main filament axis and the velocity being the line-of-sight (LOS) velocity if viewing this filament in the Galactic plane. The figure first shows these diagrams for all the gas in the $50 \mathrm{pc}^{3}$ regions. A variety of gas distributions are seen (note that to highlight individual kinematic features, independent of density, each simulation cell is plotted with an equally weighted dot). Some regions, e.g., $a, c$, and $d$, show a very broad distribution of velocities, extending over ranges of 


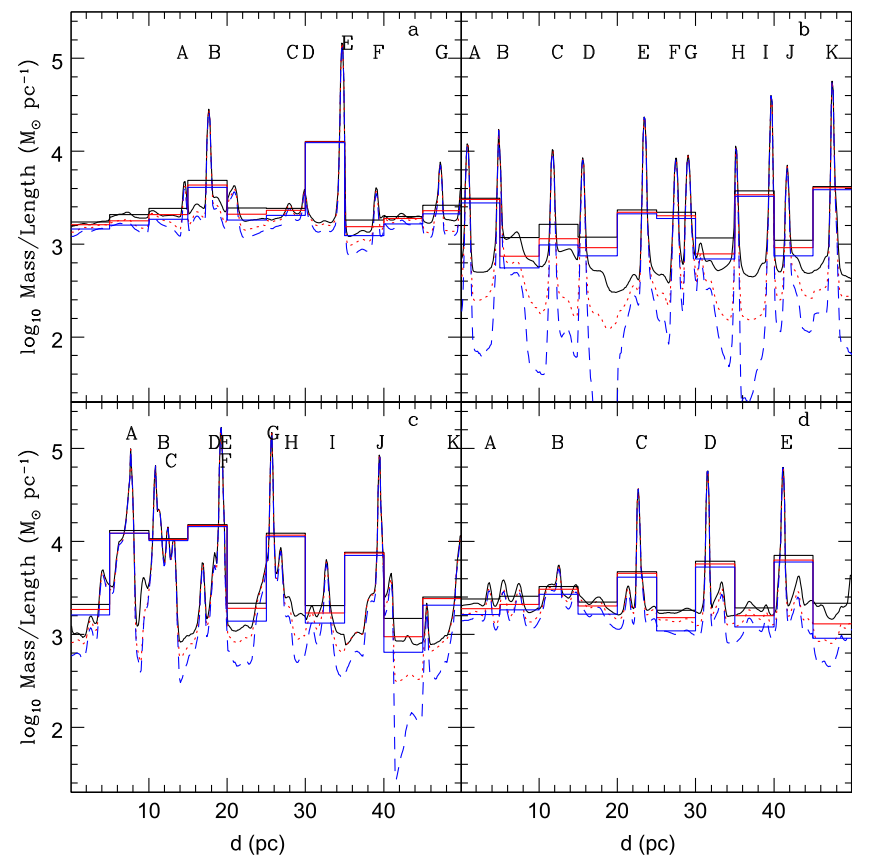

Figure 8. Mass per unit length profiles along filaments a-d, calculated in $\sim 0.1 \mathrm{pc}$ wide strips perpendicular to the outer filament (black), inner filament (red), and inner filament where $n_{\mathrm{H}}>10^{3} \mathrm{~cm}^{-3}$ (blue). The mean values for the 10 strips are also plotted in histogram form. Individual identified clumps (see text) are also labeled as "A, B, C, etc." Sometimes different clumps can have similar longitudinal coordinates but are separated laterally, as in clumps D, E, and $\mathrm{F}$ in filament $c$.

$\sim 30 \mathrm{~km} \mathrm{~s}^{-1}$ or more. Filament $b$ has a narrower range. Within each region, more coherent structures can be seen in $p-v$ space, including those that correspond to the identified filaments, which are distinguished in the second row of the figure (outer filament in black, inner in red). However, again, there are a variety of velocity structures exhibited by the different filaments, with $a, c$, and $d$ again being more disordered. This is consistent with the velocity fields shown in Figure 5.

The third row shows the near side of the outer filament strips in red and the far side in blue. Thus, if we were seeing infall to the filament, we would expect the near-side, red material to be at larger, redshifted velocities than the far-side, blue material. This kinematic feature is seen in some regions of all the filaments, although is perhaps less evident in $b$. To more clearly illustrate such effects, in Figures 11-14 we also show the velocity histograms (equivalent to optically thin spectra of an idealized tracer of material at all densities) of the outer, inner, and dense filaments in the 10 strips, also separating near- and far-side material. The disordered kinematics, i.e., different velocity distributions between near and far sides, are evident in many cases. However, caution is needed when trying to interpret blueshifted far-side material as a signature of diffuse infall onto a filament, as such signatures can also arise from discrete clumps (which may also be infalling).

Finally, in Figure 10 we also separately show the material at $n_{\mathrm{H}}>10^{2}, 10^{3}, 10^{4}$, and $10^{5} \mathrm{~cm}^{-3}$, i.e., eventually isolating the clumps that contain the dense material that is forming or will form stars. A striking feature of many of these clumps is the large velocity dispersions, which can be significantly greater than $10 \mathrm{~km} \mathrm{~s}^{-1}$. The dynamical properties of the clumps are discussed below in Section 4.4.
As mentioned, the LOS velocity distributions (i.e., optically thin spectra) are calculated and shown in Figures 11-14 for the individual strips of filaments $a-d$. The spectra for outer, inner, and dense filaments are shown, including separation of near and far sides (for the dense filament case, the same boundaries for near and far are used as in the inner filament case, but only showing gas above $n_{\mathrm{H}}>10^{3} \mathrm{~cm}^{-3}$ ).

We utilize these spectra to evaluate the mass-weighted mean LOS velocities in the strips. These values for the 10 strips in each filament are then used to measure the best-fit global $(50 \mathrm{pc}$ scale) velocity gradient, weighting the data point from each strip equally (see Table 4). These values are $\sim 0.1-$ $0.2 \mathrm{~km} \mathrm{~s}^{-1} \mathrm{pc}^{-1}$ and do not vary much going from outer to inner to dense filament structures. On the 5 pc scales from strip to strip centers we also have nine measurements of velocity gradients, which have averages of $0.446 \pm 0.134$, $0.135 \pm 0.0923, \quad 0.553 \pm 0.446, \quad$ and $\quad 0.358 \pm 0.312$ $\mathrm{km} \mathrm{s}^{-1} \mathrm{pc}^{-1}$ for the outer filaments $a-d$, respectively (reported in the 10th row for each filament in Table 4). Here the uncertainty measurement indicates the dispersion in the values. Similar results are seen for the inner and dense filament cases. The above results indicate that on scales of $\sim 5 \mathrm{pc}$ (similar to the clump to clump separation scales) the velocity gradients are several times larger than when averaged over $50 \mathrm{pc}$ scales.

The observed Galactic $\sim 100$ pc scale filaments have global velocity gradients that are very small. Jackson et al. (2010) find $d v / d l<0.09 \mathrm{~km} \mathrm{~s}^{-1} \mathrm{pc}^{-1}$ in "Nessie," Battersby \& Bally (2012) find $d v / d r<0.05 \mathrm{~km} \mathrm{~s}^{-1} \mathrm{pc}^{-1}$ in their $80 \mathrm{pc}$ long cloud, and Ragan et al. (2014) measure $d v / d r \simeq 0.06 \mathrm{~km} \mathrm{~s}^{-1} \mathrm{pc}^{-1}$ as an average of the seven filaments in their sample. On smaller scales, there have been some reported measurements of velocity gradients within IRDCs. For example, Henshaw et al. (2014) find global velocity gradients of $0.08,0.07$, and $0.30 \mathrm{~km} \mathrm{~s}^{-1} \mathrm{pc}^{-1}$ in several sub-filaments in IRDC $\mathrm{H}$ measured on $\sim 2 \mathrm{pc}$ scales based on centroid velocities of the dense gas tracer $\mathrm{N}_{2} \mathrm{H}^{+}(1-0)$. Larger local gradients $\sim 1.5-2.5 \mathrm{~km} \mathrm{~s}^{-1} \mathrm{pc}^{-1}$ can be present on sub-parsec scales.

The simulated filament $b$ comes closest to matching the above observed values, while $a, c$, and $d$ have $50 \mathrm{pc}$ scale gradients that are several times larger. More detailed comparison, e.g., of gradients on smaller $\lesssim 10$ pc scales, is warranted, but this initial study indicates that our simulated filaments have much more disturbed kinematics on large $\gtrsim 10$ pc scales than the observed Galactic long filaments.

\subsection{Filament and Clump Dynamics}

The simulated filaments have formed by large-scale collapse of self-gravitating "GMCs," in which collapse is unable to be resisted by magnetic fields or local feedback from star formation. Some resistance to collapse is provided by the turbulent and shearing motions present in and around the clouds due to their galactic environment. By $4 \mathrm{Myr}$ after the beginning of the simulation, the four filaments that we have chosen for analysis are in various stages of collapse and fragmentation. Here we assess their dynamical state, i.e., how close are they to virial equilibrium?

We carry out a filamentary virial analysis for each filament strip following Fiege \& Pudritz (2000), who derived the following equation satisfied by pressure-confined, nonrotating, self-gravitating, filamentary (i.e., lengths $\gg$ widths) clouds that 


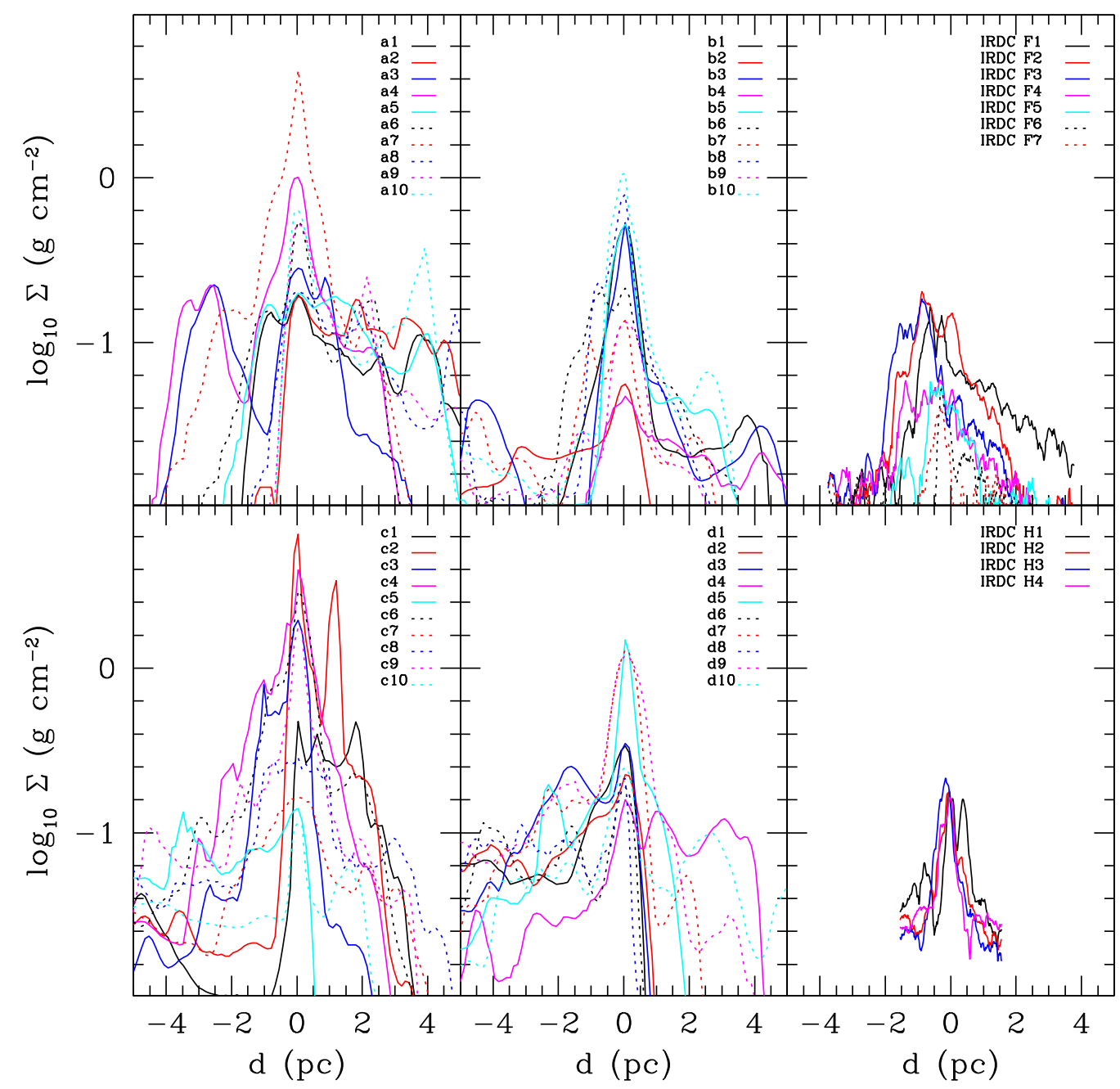

Figure 9. Mean lateral $\Sigma$ profiles perpendicular to the axes of filaments $a-d$, calculated in each of the 10 outer filament strip regions, i.e., 10 pc wide (perpendicular to the filament long axis), $10 \mathrm{pc}$ deep, and averaging over a $5 \mathrm{pc}$ length along the filament long axis. In the right column we show the profiles across two filamentary IRDCs, using the strip locations and sizes from Hernandez et al. (2011).

are in virial equilibrium:

$$
\frac{P_{e}}{P_{f}}=1-\frac{m_{f}}{m_{\mathrm{vir}}}\left(1-\frac{\mathcal{M}_{l}}{\left|W_{l}\right|}\right) .
$$

Here $P_{e}$ is the external envelope pressure at the filament surface, $P_{f}=\rho_{f} \sigma_{f}^{2}$ is the average total pressure in the filament, $m_{f}$ is the filament mass per unit length, $m_{\text {vir }} \equiv 2 \sigma_{f}^{2} / G$ is the filament virial mass per unit length, $\mathcal{M}_{f}$ is the magnetic energy per unit length, and $W_{f}=-m_{f}^{2} G$ is the gravitational energy per unit length.

For each filament strip, for the cases of outer, inner, and dense filament regions, we measure the mass-weighted 1D velocity dispersion in the direction of observation that is in the galactic plane and orthogonal to the filament main axis. These values are listed in Table 3, along with the dispersions measured in other directions. The velocity dispersions are supersonic, with Mach numbers of about 20 for gas that is cooled to about $10 \mathrm{~K}$. Note that the $z$-direction velocity dispersions are similar in size to those in directions in the Galactic plane (unlike GMC motions), indicating that approximately isotropic support may be possible from these turbulent motions.

We also measure the velocity dispersions in the surrounding envelope regions, which, together with the density of these regions, allows us to assess the turbulent pressure that acts as a surface term, $P_{e}=\rho_{e} \sigma_{e}^{2}$, affecting the virial equilibrium of the filaments. Here we choose to measure the volume-averaged density in the envelope region and the mass-weighted velocity dispersion, which is comparable to the quantities derived observationally for density (from extinction maps) and velocity dispersion from ${ }^{13} \mathrm{CO}$ and $\mathrm{C}^{18} \mathrm{O}$ spectra (e.g., Hernandez \& Tan 2011; Hernandez et al. 2012). We discuss, below, the effects of these choices on our results.

In Figure 15, we compare the simulated filament strips with the non-magnetic $\left(\mathcal{M}_{f}=0\right)$ form of Equation (2). For each strip we show the outer, inner, and dense filament results connected by a line. In general, the filaments have $0.1 \gtrsim m_{f} / m_{\text {vir }} \lesssim 1$, but this is not typically due to higher envelope pressure. Thus, most filament strip regions appear to not yet be virialized, having very disordered kinematics due to infall motions and motions associated with dense, spheroidal clumps that have already fragmented from the filament, discussed below. Only in a few regions of filaments $b$ (outer) 


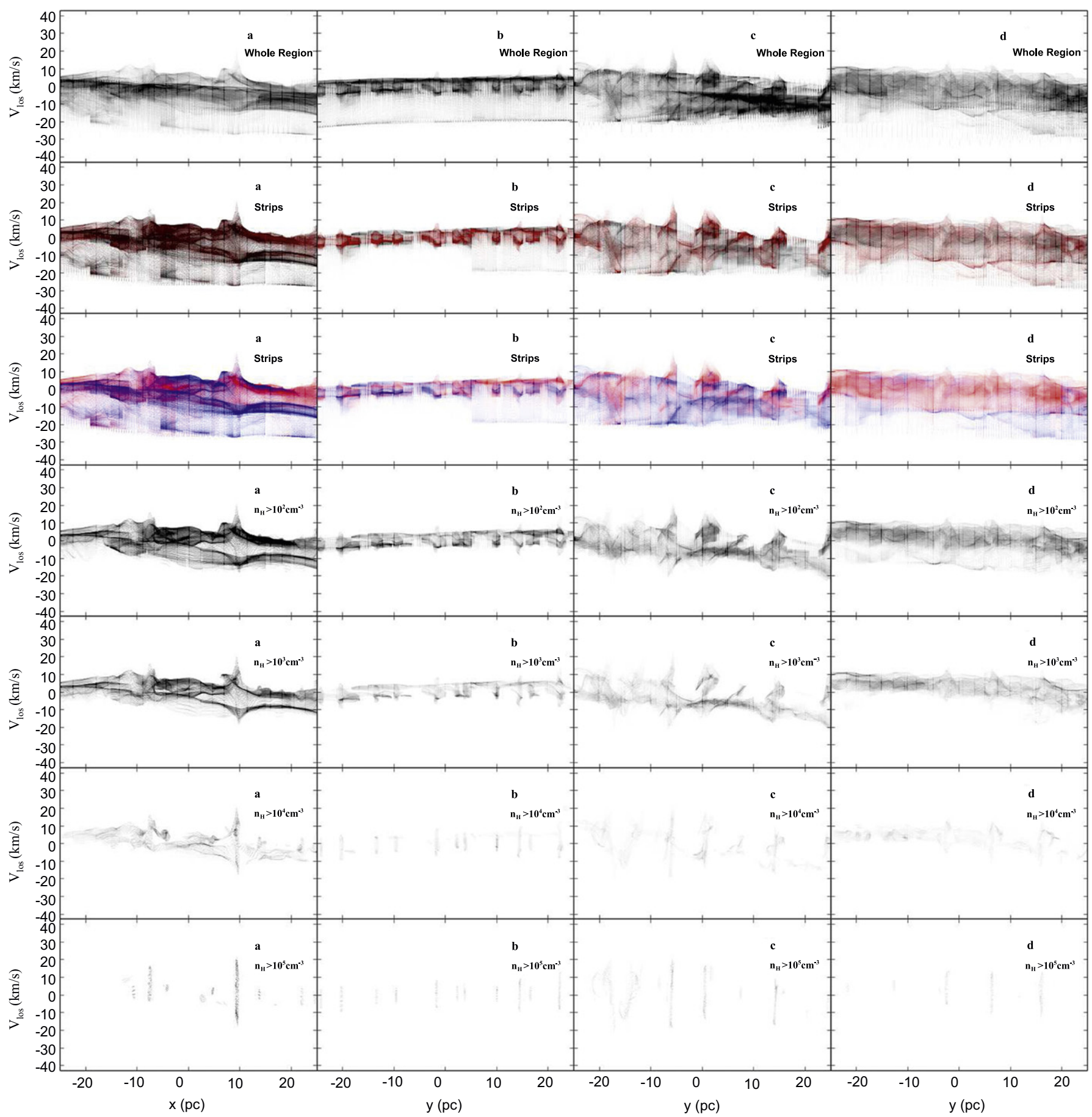

Figure 10. Position-velocity diagrams for filaments $a-d$ (left to right), where the position coordinate has been chosen to be along the main axis of the filament, i.e., $x$ direction for $a$ and $y$-direction for $b-d$. The top row shows all the gas in the $50 \mathrm{pc}^{3}$ regions. For clarity of highlighting kinematic structures, each simulation cell is marked by a simple dot (thus the AMR gridding appears as vertical stripes). The second row shows only the material in the filament strips, with outer (10 pc-wide) strips in black and inner ( 5 pc-wide) strips in red. The third row shows material on the near side of the outer filament in red and the far side in blue. The fourth, fifth, sixth, and seventh rows show only gas at $n_{\mathrm{H}}>10^{2}, 10^{3}, 10^{4}$, and $10^{5} \mathrm{~cm}^{-3}$, respectively.

and $d$ (dense) are conditions closer to filamentary virial equilbrium. Note that filament $b$ 's inner region is highly fragmented into clumps, and so when defining the envelope material around the "dense" filament material, we derive relatively low values leading to values of $P_{e} / P_{f}$ much lower than expected by filamentary virial equilbrium. Filament $d$, being relatively less fragmented into clumps, shows inner region conditions that are closer to virial equilibrium.
Here we examine more quantitatively how fragmentation of filaments into clumps that are themselves approximately virialized can lead to small values of $m_{f} / m_{\text {vir }}$ for the filament. Consider the case when a fraction, $\epsilon_{\mathrm{cl}}$, of the filament strip mass, $M$, has condensed into a virialized clump of radius $R_{\mathrm{cl}}$ and velocity dispersion $\sigma_{\mathrm{cl}}$, which is given by $\sigma_{\mathrm{cl}}^{2}=G \epsilon_{\mathrm{cl}} M /\left(5 R_{\mathrm{cl}}\right)$ (note that corrections for ellipticity and 


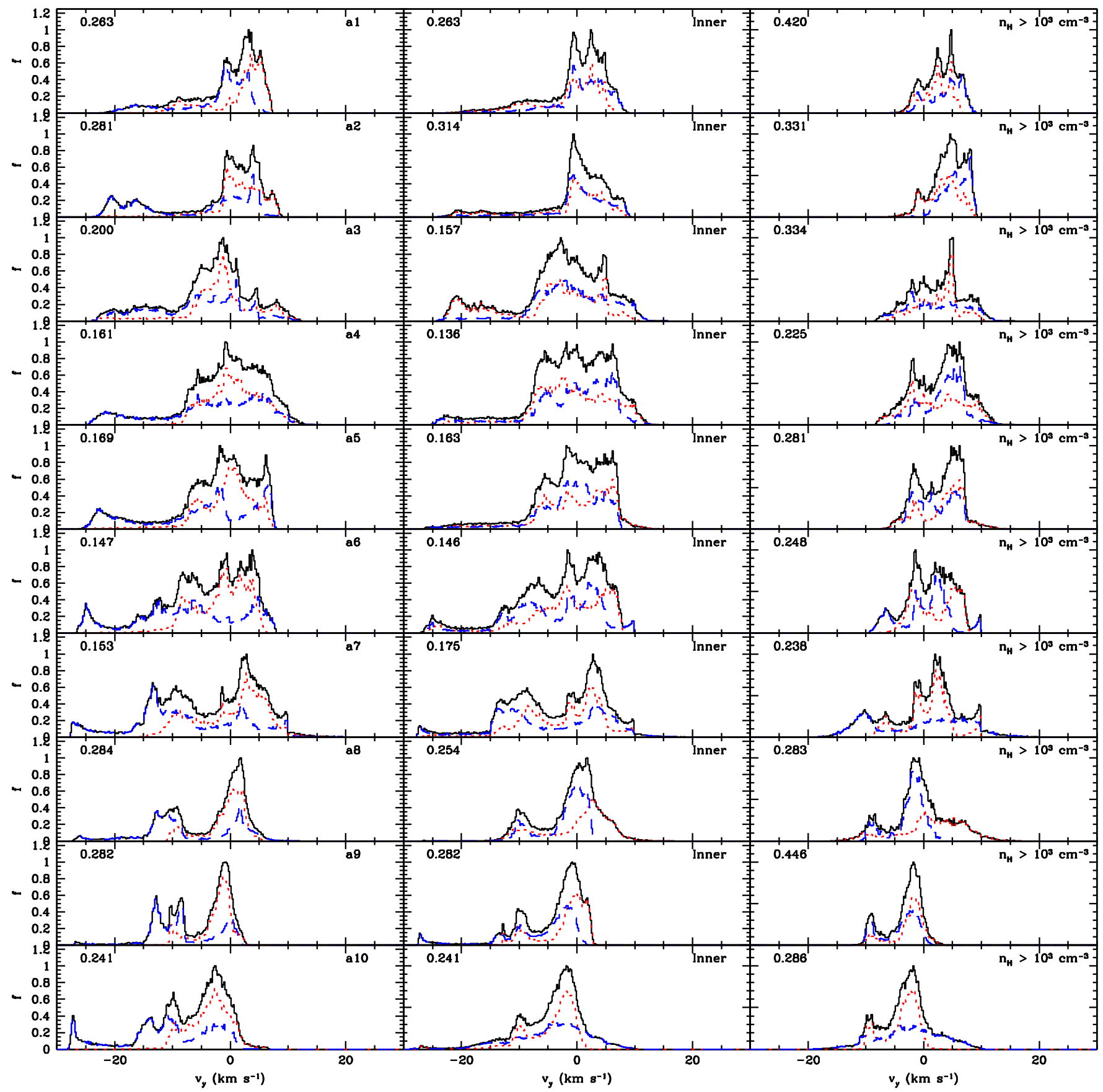

Figure 11. LOS velocity spectra for each filament strip (1 to 10, from top to bottom) for filament $a$. Left, middle, and right columns show the outer, inner, and dense filaments, respectively (see text for definitions). Total spectra are shown with black solid lines. The filament strips are also divided into near- (red dotted) and far-side (blue dashed) side regions with respect to each strip's center of mass. The normalization factor is given in the top left corner of each panel in units of $\mathrm{g} \mathrm{cm}^{-2} /\left(\mathrm{km} \mathrm{s}^{-1}\right)$.

central concentration of the clump are typically modest; Bertoldi \& McKee 1992). The velocity dispersion expected from the filamentary virial theorem is given by $\sigma_{f}^{2}=G m_{f} / 2=G M /(2 L)$, where $L$ is the length of the strip, i.e., $5 \mathrm{pc}$ in the cases considered here. Then,

$$
\frac{\sigma_{\mathrm{cl}}^{2}}{\sigma_{f}^{2}}=\frac{2 L}{5 R_{\mathrm{cl}}} \epsilon_{\mathrm{cl}}
$$

and so with $\sigma_{\mathrm{tot}}^{2}=\epsilon_{\mathrm{cl}} \sigma_{\mathrm{cl}}^{2}+\left(1-\epsilon_{\mathrm{cl}}\right) \sigma_{f}^{2}$, then

$$
\frac{\sigma_{\mathrm{tot}}^{2}}{\sigma_{f}^{2}}=\left(1-\epsilon_{\mathrm{cl}}\right)+\frac{2}{5} \frac{L}{R_{\mathrm{cl}}} \epsilon_{\mathrm{cl}}^{2} \text {. }
$$

If $\epsilon_{\mathrm{cl}} \gtrsim 5 R_{\mathrm{cl}} /(2 L)$, then the total velocity dispersion begins to increase above that expected from the filamentary virial theorem. We will see below that identified clumps have typical radii of $\sim 0.5 \mathrm{pc}$, so then the critical efficiency for increasing velocity dispersion is $\simeq 0.25\left(R_{\mathrm{cl}} / 0.5 \mathrm{pc}\right)$. 


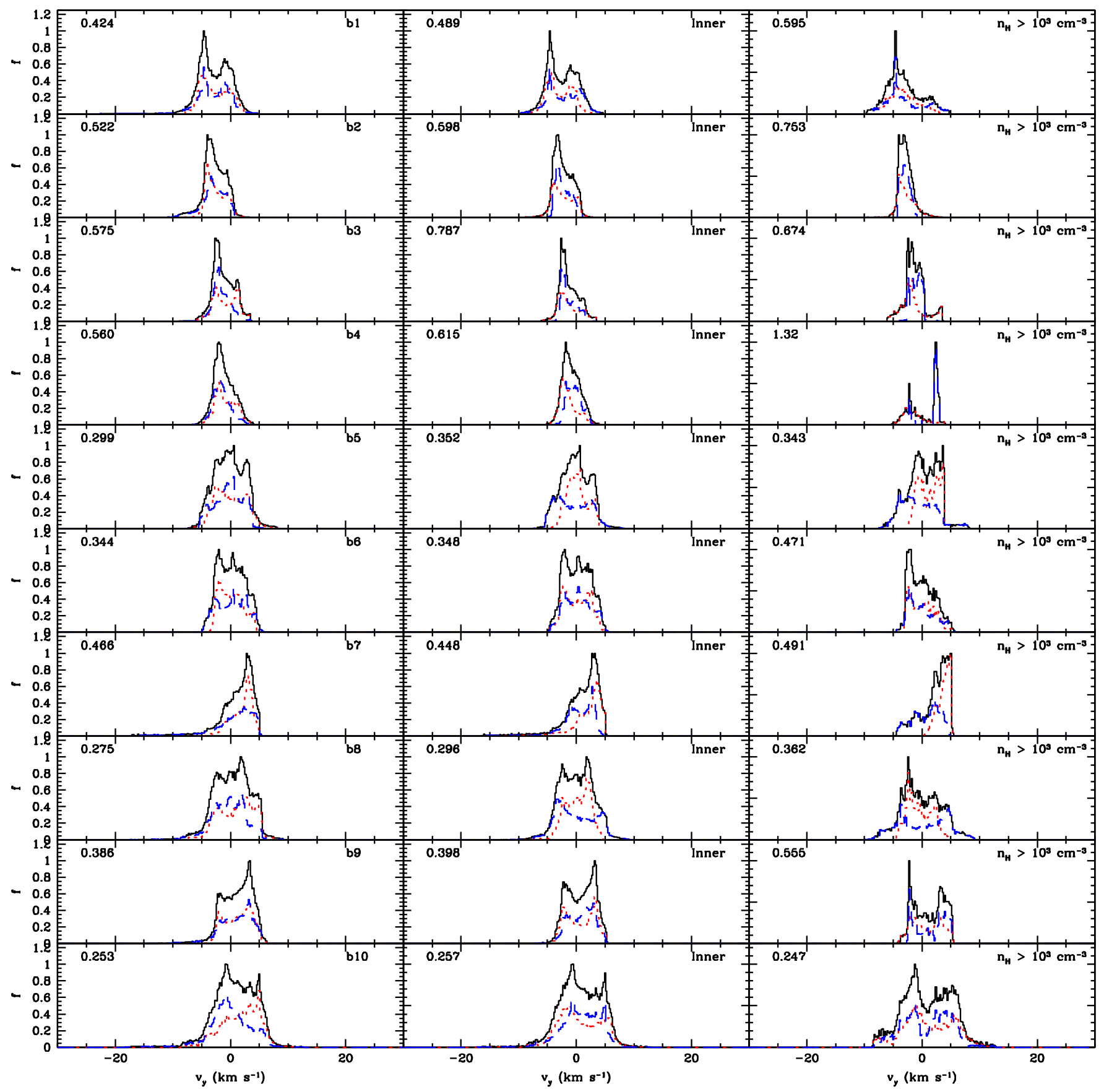

Figure 12. Same as Figure 11, but now for filament $b$.

For example, strip $a 7$, which has a relatively small value of $m_{f} / m_{\text {vir }} \simeq 0.25$, has a $5 \times 10^{4} M_{\odot}$ clump, so that $\epsilon_{\mathrm{cl}} \simeq 0.8$. It has a radus of about $0.7 \mathrm{pc}$ and a mean velocity dispersion in the $y$-direction of $11 \mathrm{~km} \mathrm{~s}^{-1}$ (it has a virial parameter of $\alpha_{\mathrm{vir}}=$ 2.0). The total velocity dispersion for the $a 7$ strip is $10.2 \mathrm{~km} \mathrm{~s}^{-1}$. However, with its mass per unit length of $1.28 \times 10^{4} M_{\odot} \mathrm{pc}^{-1}$, one would expect a virialized velocity dispersion of only $5.2 \mathrm{~km} \mathrm{~s}^{-1}$. This difference is explained by the clump-boosting factor for velocity dispersion squared predicted by Equation (4) of about 2.0, i.e., a factor of 1.4 for the velocity dispersion. Since the clump itself is moderately supervirial, this explains why the actual velocity dispersion increase is larger. So when placing the $a 7$ strip on the FiegePudritz diagram, we expect $m_{l} / m_{\text {vir }}$ to be $\simeq 0.25$. Similarly, the dominance within the filament strip of spheroidal clump dynamics causes a very low value of $P_{e} / P_{f}$.

Given the difficulties of resolving clumps and their fragmentation from the filament, we have focused mostly on filament dynamics for comparison of simulation results with observations. However, for completeness, here we give a brief assessment of the dynamical state of the identified clumps. For each of the clumps identified by the threshold density of $10^{5} \mathrm{~cm}^{-3}$, we measure the mass in the cells, the mass-weighted velocity dispersion about the center-of-mass velocity, and the mean half-mass radius, $R_{1 / 2}$, in each of the $x$-, $y$-, and $z$ directions, and the average value. We then evaluate the virial parameter, $\alpha_{\mathrm{vir}}=5 \sigma^{2} R_{1 / 2} /\left(G M_{1 / 2}\right)$ (Bertoldi \& McKee 1992), at this half-mass scale. The results are summarized in Table 5. 


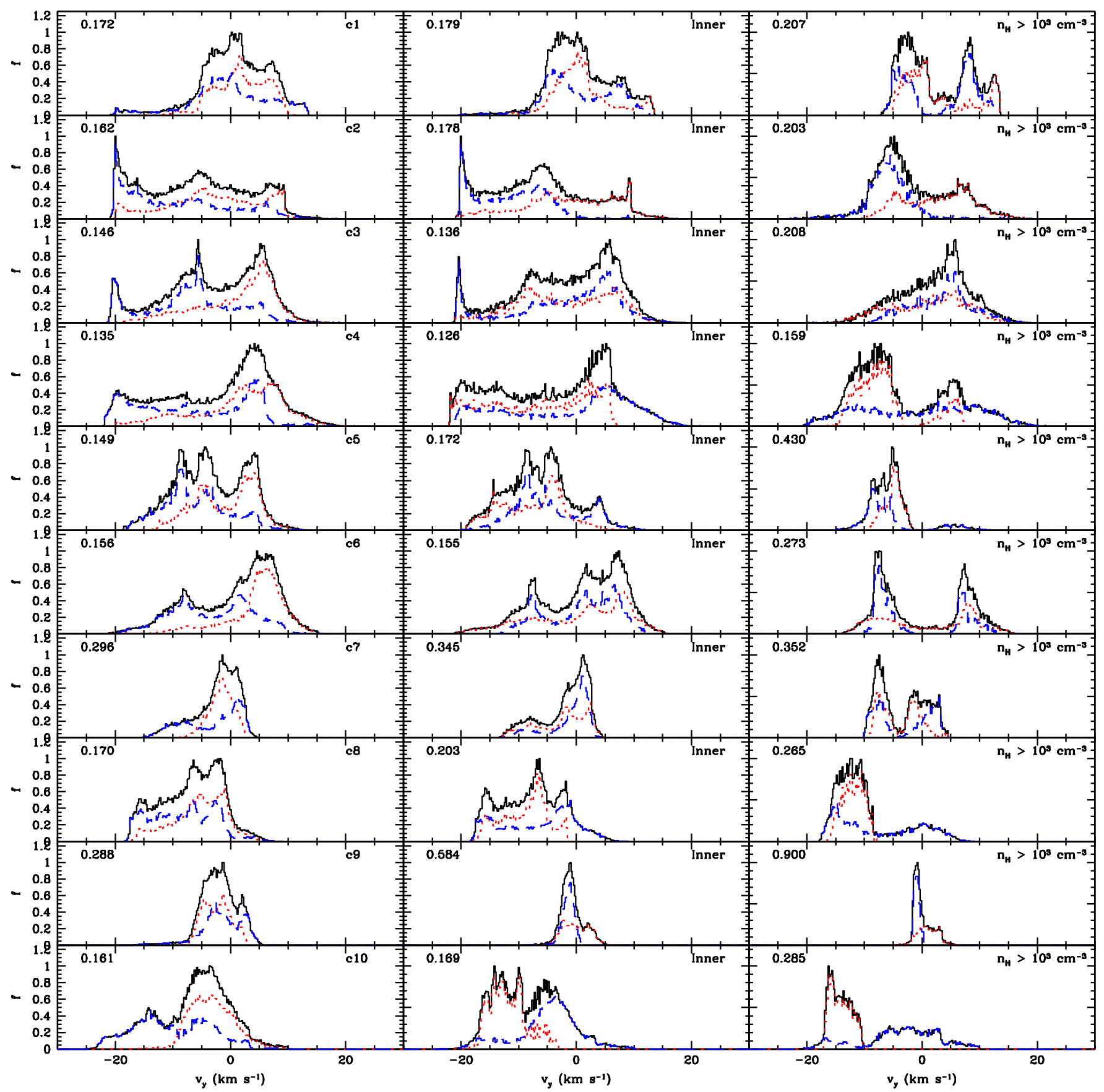

Figure 13. Same as Figure 11, but now for filament $c$.

The clumps tend to have virial parameters $\simeq 1$, and almost always $<2$, indicating they are gravitationally bound and perhaps moderately supervirial (although a contribution from surface pressure would also raise the virialized value of $\alpha_{\mathrm{vir}}$ above unity). We tentatively conclude, with the caveat that increased numerical resolution is needed, that these clump structures are much closer to virial equilibrium than their parental filaments, which is to be expected given their much shorter dynamical times.

\section{DISCUSSION AND CONCLUSIONS}

We have continued the study begun in Van Loo et al. (2013), following the evolution of a kiloparsec-scale patch of a galactic disk extracted from a global disk simulation down to $\sim 0.1 \mathrm{pc}$ resolution. We have followed collapse for $4 \mathrm{Myr}$, which is ample time for the formation of dense filaments and clumps from the initial GMCs and, in the run allowing star formation, for significant star formation activity.

The main goal has been to study the detailed structural, kinematic, and dynamical properties of filamentary clouds that are in relatively early stages of collapse at $t=4 \mathrm{Myr}$. Even in the run where star formation is allowed (above the threshold density of $n_{\mathrm{H}}=10^{6} \mathrm{~cm}^{-3}$ ), almost no star formation has yet occurred in our sample of filaments. These properties have been compared to those of observed filamentary IRDCs, which are also thought to be in a relatively early stage of collapse and star formation. Note that IRDCs are thus thought to be 


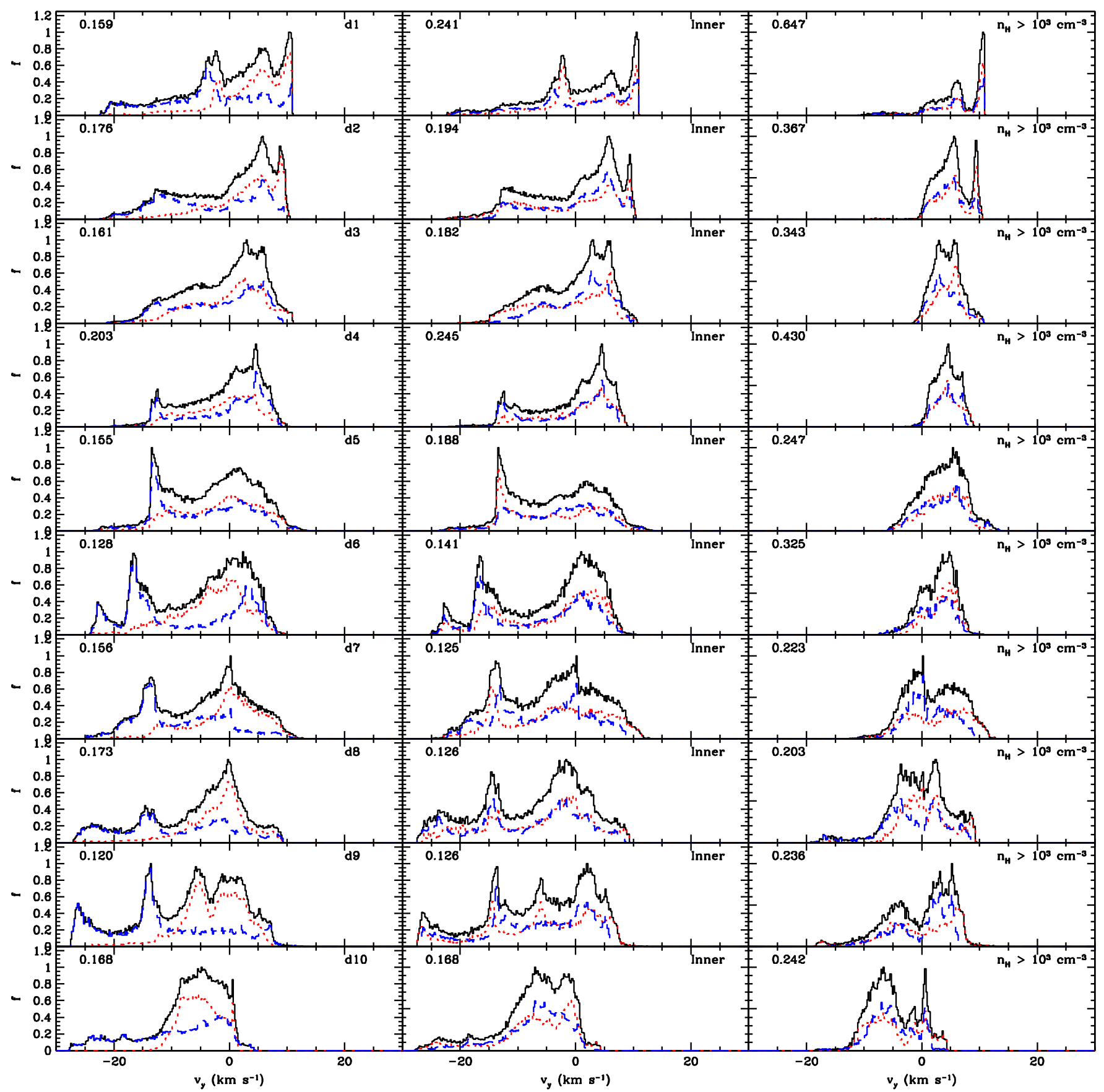

Figure 14. Same as Figure 11, but now for filament $d$.

relatively unaffected by internal feedback from star formation, simplifying the comparison of simulation with observation by avoiding having to simulate star formation feedback, which requires much higher resolution or more uncertain sub-grid models.

Our main conclusion is that the simulated filaments, which are forming from global collapse of gravitationally unstable GMCs mediated by galactic shear driven turbulence, show significant differences from observed IRDCs. The filaments and their surrounding $50 \mathrm{pc}$ scale regions often have dense gas mass fractions, e.g., at $\Sigma>1 \mathrm{~g} \mathrm{~cm}^{-2}$, that are larger than even the most extreme IRDCs. The simulated filaments show large dispersions in their mass per unit lengths, caused by their fragmentation into dense clumps. The simulated filaments have more disordered kinematics, including velocity gradients as measured on 50 and $5 \mathrm{pc}$ length scales. These more disordered kinematics equate to larger velocity dispersions than expected of virialized filaments.

The implications of these results are that IRDCs do not form by fast global collapse of gravitationally unstable GMCs. Mediation, regulation, and slowing of collapse by dynamically strong magnetic fields seem to be the most promising mechanism by which to reconcile simulations with observed IRDCs. This scenario is given support by the recent observational results of Pillai et al. (2015), who infer $\sim \mathrm{mG}$ magnetic fields and sub-Alfvénic turbulence in IRDCs G11.11-0.12 and G0.253+0.016 from the ordered orientations of sub-mm dust emission polarization vectors. Lower $(0.5 \mathrm{pc})$ 
Table 4

Filament Velocity Gradients

\begin{tabular}{|c|c|c|}
\hline Filament & $\begin{array}{c}\bar{v}_{\text {los }} \\
\left(\mathrm{km} \mathrm{s}^{-1}\right)\end{array}$ & $\begin{array}{c}d v_{\mathrm{los}} / d l \\
\left(\mathrm{~km} \mathrm{~s}^{-1} \mathrm{pc}^{-1}\right)\end{array}$ \\
\hline$a$ & $-0.223,0.242,0.531$ & $0.224,0.2230 .228$ \\
\hline$a 1$ & $2.85,2.92,3.11$ & $0.140,0.210,0.220$ \\
\hline$a 2$ & $3.55,3.97,4.22$ & $0.206,0.204,0.148$ \\
\hline a3 & $2.52,2.95,3.48$ & $0.278,0.334,0.296$ \\
\hline$a 4$ & $3.91,4.62,4.96$ & $0.688,0.618,0.384$ \\
\hline$a 5$ & $0.471,1.53,1.98$ & $0.384,0.514,0.470$ \\
\hline$a 6$ & $-1.45,-1.04,-0.371$ & $0.678,0.616,0.526$ \\
\hline$a 7$ & $1.94,2.04,2.26$ & $1.30,1.16,1.20$ \\
\hline$a 8$ & $-4.57,-3.74,-3.75$ & $0.266,0.404,0.396$ \\
\hline$a 9$ & $-5.90,-5.76,-5.73$ & $0.0700,0.138,0.176$ \\
\hline$a 10$ & $-5.55,-5.07,-4.85$ & {$[0.446,0.466,0.448]^{\mathrm{a}}$} \\
\hline$b$ & $-0.138,-0.237,-0.292$ & $0.0868,0.0804,0.0810$ \\
\hline$b 1$ & $-2.43,-2.31,-2.29$ & $0.0460,0.0100,0.0280$ \\
\hline$b 2$ & $-2.20,-2.36,-2.43$ & $0.313,0.242,0.270$ \\
\hline$b 3$ & $-0.636,-1.15,-1.08$ & $0.0492,0.0810,0.0840$ \\
\hline$b 4$ & $-0.390,-0.745,-0.660$ & $0.0474,0.169,0.106$ \\
\hline$b 5$ & $-0.153,0.102,-0.130$ & $0.153,0.0988,0.151$ \\
\hline$b 6$ & $0.612,0.596,0.624$ & $0.216,0.147,0.159$ \\
\hline$b 7$ & $1.69,1.33,1.42$ & $0.183,0.187,0.202$ \\
\hline$b 8$ & $0.773,0.396,0.411$ & $0.137,0.0580,0.0640$ \\
\hline$b 9$ & $1.46,0.686,0.731$ & $0.0680,0.0808,0.0678$ \\
\hline$b 10$ & $1.12,1.09,1.07$ & {$[0.135,0.119,0.126]^{\mathrm{a}}$} \\
\hline$c$ & $-1.16,-0.584,-2.93$ & $0.224,0.208,0.210$ \\
\hline$c 1$ & $4.56,5.41,2.90$ & $0.664,0.764,0.770$ \\
\hline$c 2$ & $1.24,1.59,-0.952$ & $0.390,0.356,0.452$ \\
\hline$c 3$ & $3.19,3.37,1.31$ & $0.344,0.360,0.512$ \\
\hline$c 4$ & $1.47,1.57,-1.25$ & $1.13,1.16,0.748$ \\
\hline$c 5$ & $-4.19,-4.22,-4.99$ & $1.09,1.15,0.708$ \\
\hline$c 6$ & $1.27,1.52,-1.45$ & $1.04,0.912,0.704$ \\
\hline$c 7$ & $-3.95,-3.04,-4.97$ & $0.0480,0.232,0.558$ \\
\hline$c 8$ & $-4.19,-4.20,-7.76$ & $0.256,0.400,0.754$ \\
\hline$c 9$ & $-5.47,-2.20,-3.99$ & $0.00600,0.688,0.828$ \\
\hline$c 10$ & $-5.50,-5.64,-8.13$ & {$[0.553,0.669,0.671]^{\mathrm{a}}$} \\
\hline$d$ & $1.09,0.970,2.20$ & $0.172,0.198,0.221$ \\
\hline$d 1$ & $3.83,3.97,6.21$ & $0.0280,0.0260,0.150$ \\
\hline$d 2$ & $3.97,4.10,5.46$ & $0.0900,0.0740,0.162$ \\
\hline$d 3$ & $3.52,3.73,4.65$ & $0.188,0.152,0.0840$ \\
\hline$d 4$ & $2.58,2.97,4.23$ & $0.0540,0.00200,0.140$ \\
\hline$d 5$ & $2.85,2.96,3.53$ & $0.549,0.630,0.224$ \\
\hline$d 6$ & $0.106,-0.191,2.41$ & $0.263,0.336,0.0120$ \\
\hline$d 7$ & $1.42,1.49,2.35$ & $0.764,0.856,0.522$ \\
\hline$d 8$ & $-2.40,-2.79,-0.260$ & $0.419,0.511,0.192$ \\
\hline$d 9$ & $-0.306,-0.235,-1.22$ & $0.869,1.21,0.824$ \\
\hline$d 10$ & $-4.65,-6.30,-5.34$ & {$[0.358,0.422,0.257]^{\mathrm{a}}$} \\
\hline
\end{tabular}

${ }^{\text {a }}$ Average of the nine strip-to-strip values.

resolution simulations of the same initial conditions as our study, but including magnetic fields, have been recently presented by Van Loo et al. (2015, Paper II). A future goal is to extend these to $0.1 \mathrm{pc}$ or higher resolution to be able to examine the affect of magnetic field on filament structure, kinematics, and dynamics.

A more general output of this paper has been the presentation of a range of metrics of cloud, especially filamentary cloud, properties related to structure, kinematics,

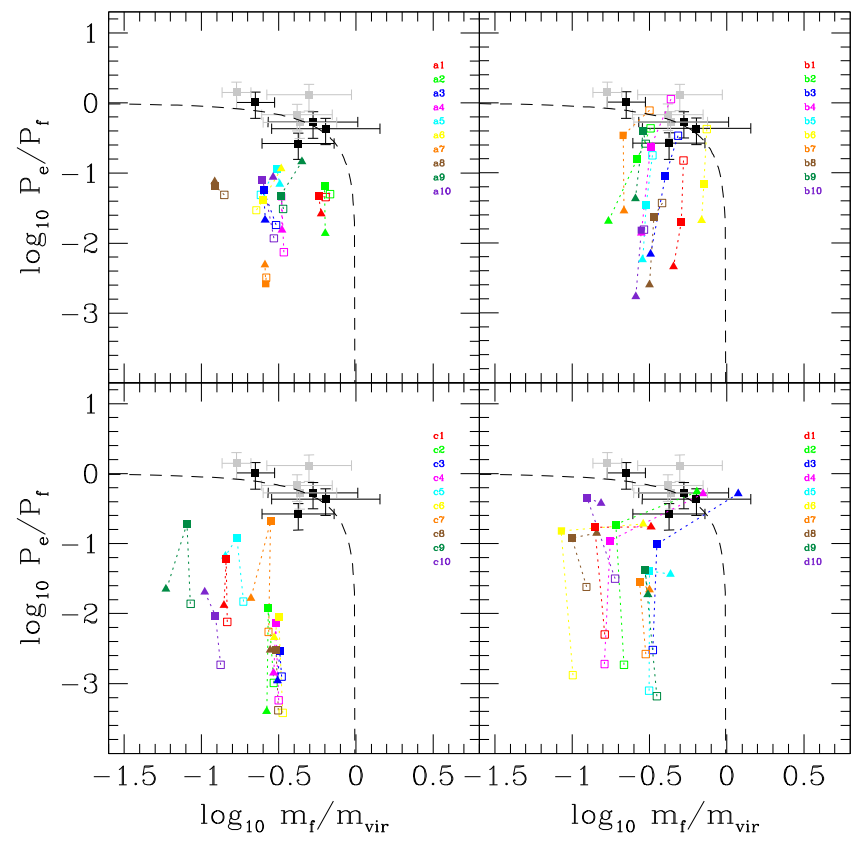

Figure 15. Filamentary virial analysis for filaments $a$ (top left), $b$ (top right), $c$ (bottom left), and $d$ (bottom right). Each panel shows the ratio of envelope, $P_{e}$, and filament, $P_{f}$, pressures vs. the ratio of filament mass per unit length, $m_{f}$, to the virial mass per unit length, $m_{\mathrm{vir}}$, for outer (open squares), inner (solid squares), and dense (solid triangles) filament regions. The case for no magnetic field support is shown as a dashed line (Fiege \& Pudritz 2000). Points from IRDC H (Hernandez et al. 2012) are plotted as gray (inner filament) and black (outer filament) points with error bars.

and dynamics. These properties are presented for multi-phase ISM, especially molecular, clouds evolving under pure selfgravitating hydrodynamics, i.e., without inclusion of magnetic fields or star formation feedback (although with the focus on nearly starless clouds, this feedback is expected to be limited). These cloud metrics include structural properties on 25 and $50 \mathrm{pc}$ region scales, including PDFs of mass surface density and the fraction of gas above $1 \mathrm{~g} \mathrm{~cm}^{-2}$. Structural properties of filaments include mass per unit length, dispersion in mass per unit length, filament and envelope densities, and lateral widths. Kinematic properties include filament and envelope velocity dispersions, comparison of mass per unit lengths with virial mass per unit lengths, external to internal pressures, and velocity gradients on various scales. Standard kinematic and dynamical properties of clumps, forming in the filaments, have also been presented. These properties can all be compared to observed clouds, especially those of IRDCs, as well as future simulations that include additional physics, especially magnetic fields and star formation feedback.

We thank Paola Caselli and Sam Falle for useful discussions, and Elizabeth Tasker for providing the initial conditions of our simulations. M.J.B. acknowledges support from the STARFORM Sinergia project funded by the Swiss National Science Foundation. S.v.L. acknowledges support from the Theory Postdoctoral Fellowship from UF Department of Astronomy and College of Liberal Arts and Sciences and from the SMA Postdoctoral Fellowship of the Smithsonian Astrophysical Observatory. J.C.T. acknowledges support from NSF CAREER grant AST-0645412; NASA Astrophysics Theory and Fundamental Physics grant ATP09-0094; NASA Astrophysics Data Analysis Program ADAP10-0110. Resources 
Table 5

Clump Sample

\begin{tabular}{|c|c|c|c|c|c|c|c|c|c|c|c|}
\hline Clump & Strip & $\begin{array}{c}x_{c}, y_{c}, z_{c} \\
(\mathrm{pc})\end{array}$ & $\begin{array}{c}\text { Mass } \\
\left(10^{4} M_{\odot}\right)\end{array}$ & $\begin{array}{c}\text { Radius } \\
\quad(\mathrm{pc})\end{array}$ & $\begin{array}{c}\bar{v}_{x} \\
\left(\mathrm{~km} \mathrm{~s}^{-1}\right)\end{array}$ & $\begin{array}{c}\bar{v}_{y} \\
\left(\mathrm{~km} \mathrm{~s}^{-1}\right)\end{array}$ & $\begin{array}{c}\bar{v}_{z} \\
\left(\mathrm{~km} \mathrm{~s}^{-1}\right)\end{array}$ & $\begin{array}{c}\bar{\sigma}_{v_{x}} \\
\left(\mathrm{~km} \mathrm{~s}^{-1}\right)\end{array}$ & $\begin{array}{c}\bar{\sigma}_{v_{y}} \\
\left(\mathrm{~km} \mathrm{~s}^{-1}\right)\end{array}$ & $\begin{array}{c}\bar{\sigma}_{v_{z}} \\
\left(\mathrm{~km} \mathrm{~s}^{-1}\right)\end{array}$ & $\alpha_{\mathrm{vir}}$ \\
\hline$a_{A}$ & $a 3$ & $469,541,2.56$ & 0.101 & 0.325 & 0.864 & 1.29 & 0.893 & 1.63 & 2.09 & 1.76 & 1.63 \\
\hline$a_{B}$ & $a 4$ & $473,541,2.53$ & 1.03 & 0.485 & -4.55 & 6.24 & -2.06 & 4.41 & 5.98 & 5.52 & 1.95 \\
\hline$a_{C}$ & $a 6$ & $483,544,0.553$ & 0.0651 & 0.299 & 4.25 & -2.18 & 1.01 & 1.14 & 1.21 & 0.542 & 0.784 \\
\hline$a_{D}$ & $a 7$ & $485,542,2.21$ & 0.0645 & 0.311 & 5.63 & 2.04 & 0.525 & 1.23 & 1.10 & 1.35 & 0.675 \\
\hline$a_{E}$ & $a 7$ & $490,543,1.36$ & 4.90 & 0.713 & 1.33 & 2.45 & -0.660 & 5.43 & 11.0 & 11.1 & 2.03 \\
\hline$a_{F}$ & $a 8$ & $494,539,3.26$ & 0.0681 & 0.298 & -0.952 & 0.0854 & 1.50 & 1.30 & 1.58 & 1.63 & 1.27 \\
\hline$a_{G}$ & $a 10$ & $502,539,2.25$ & 0.181 & 0.336 & 9.00 & -1.54 & 1.52 & 1.93 & 2.86 & 2.63 & 1.77 \\
\hline$b_{A}$ & $b 1$ & $624,586,2.03$ & 0.316 & 0.444 & -2.21 & -6.85 & -0.0531 & 3.14 & 1.76 & 2.57 & 1.61 \\
\hline$b_{B}$ & $b 1$ & $626,590,2.43$ & 0.621 & 0.485 & -2.15 & -6.75 & 0.0253 & 4.50 & 2.59 & 4.23 & 1.84 \\
\hline$b_{C}$ & $b 3$ & $628,597,1.99$ & 0.239 & 0.391 & -1.00 & -4.10 & 0.185 & 2.45 & 1.75 & 3.12 & 1.15 \\
\hline$b_{D}$ & $b 4$ & $628,601,1.89$ & 0.189 & 0.438 & -0.494 & -3.60 & -0.320 & 2.21 & 1.05 & 2.10 & 1.31 \\
\hline$b_{E}$ & $b 5$ & $629,608,1.51$ & 0.622 & 0.502 & 0.504 & 0.996 & -0.0556 & 4.48 & 1.92 & 4.41 & 1.88 \\
\hline$b_{F}$ & $b 6$ & $630,612,0.798$ & 0.151 & 0.362 & 1.13 & 1.70 & 1.48 & 2.45 & 1.25 & 2.06 & 1.67 \\
\hline$b_{G}$ & $b 6$ & $630,614,0.929$ & 0.200 & 0.420 & 1.41 & -1.23 & 0.758 & 2.31 & 1.17 & 1.62 & 1.30 \\
\hline$b_{H}$ & $b 8$ & $629,620,0.628$ & 0.0972 & 0.326 & -0.399 & 4.12 & -0.283 & 1.94 & 0.945 & 1.85 & 1.47 \\
\hline$b_{I}$ & $b 8$ & $629,624,0.281$ & 0.993 & 0.558 & 0.734 & 4.71 & -0.0817 & 5.52 & 1.89 & 4.94 & 1.99 \\
\hline$b_{J}$ & $b 9$ & $629,627,0.617$ & 0.141 & 0.379 & 0.451 & 0.629 & -0.538 & 2.20 & 1.20 & 1.97 & 1.51 \\
\hline$b_{K}$ & $b 10$ & $628,632,-0.771$ & 1.49 & 0.580 & 1.49 & 4.40 & 0.261 & 5.97 & 2.24 & 6.85 & 1.62 \\
\hline$c_{A}$ & $c 2$ & $631,233,15.2$ & 4.94 & 0.762 & 2.50 & 2.69 & 1.16 & 8.53 & 10.3 & 2.68 & 1.30 \\
\hline$c_{B}$ & $c 3$ & $624,237,17.3$ & 4.09 & 0.723 & 3.03 & 0.665 & -0.386 & 9.29 & 7.94 & 2.65 & 1.77 \\
\hline$c_{C}$ & $c 3$ & $626,238,17.5$ & 0.442 & 0.472 & 5.17 & -7.00 & 2.07 & 4.70 & 1.86 & 2.35 & 2.74 \\
\hline$c_{D}$ & $c 4$ & $624,243,17.0$ & 0.203 & 0.347 & 6.30 & 1.68 & 3.22 & 1.88 & 2.46 & 2.67 & 0.705 \\
\hline$c_{E}$ & $c 4$ & $621,245,18.0$ & 0.0980 & 0.329 & 4.64 & -3.91 & 7.75 & 1.74 & 1.22 & 1.47 & 1.18 \\
\hline$c_{F}$ & $c 4$ & $618,245,18.9$ & 5.68 & 0.781 & 1.57 & 0.294 & -0.0265 & 11.8 & 3.47 & 9.62 & 2.23 \\
\hline$c_{G}$ & $c 6$ & $612,252,20.9$ & 4.03 & 0.732 & 1.83 & -1.46 & -1.30 & 8.98 & 3.99 & 10.3 & 1.70 \\
\hline$c_{H}$ & $c 6$ & $611,253,20.3$ & 0.173 & 0.354 & 12.2 & -10.9 & -0.966 & 2.25 & 1.69 & 2.67 & 1.21 \\
\hline$c_{I}$ & $c 7$ & $608,259,23.3$ & 0.102 & 1.05 & -0.883 & -0.102 & 1.82 & 0.939 & 1.75 & 1.75 & 1.33 \\
\hline$c_{J}$ & $c 8$ & $602,265,25.4$ & 2.62 & 0.708 & -3.24 & 4.18 & 6.82 & 7.56 & 2.24 & 7.07 & 1.80 \\
\hline$c_{K}$ & $c 10$ & $593,276,28.8$ & 0.297 & 0.395 & -3.48 & -3.81 & -13.0 & 3.49 & 3.19 & 3.07 & 1.88 \\
\hline$d_{A}$ & $d 1$ & $782,178,6.87$ & 0.0445 & 0.286 & 3.86 & 3.39 & 0.268 & 1.24 & 0.867 & 1.34 & 1.15 \\
\hline$d_{B}$ & $d 3$ & $784,187,5.85$ & 0.105 & 0.357 & 5.32 & 4.38 & 0.232 & 1.65 & 1.48 & 1.75 & 1.07 \\
\hline$d_{C}$ & $d 5$ & $787,198,6.54$ & 1.16 & 0.533 & 3.95 & 4.38 & 1.09 & 6.06 & 3.58 & 5.87 & 1.96 \\
\hline$d_{D}$ & $d 7$ & $787,206,7.12$ & 1.76 & 0.575 & 1.89 & 4.69 & 0.0727 & 7.02 & 3.77 & 7.59 & 1.87 \\
\hline$d_{E}$ & $d 9$ & $784,216,7.87$ & 2.03 & 0.600 & 0.152 & 3.70 & 0.186 & 6.92 & 3.82 & 8.44 & 1.64 \\
\hline
\end{tabular}

supporting this work were provided by the University of Florida Research Computing and the NASA High-End Computing (HEC) Program through the NASA Advanced Supercomputing (NAS) Division at Ames Research Center.

\section{REFERENCES}

Arzoumanian, D., André, Ph., Didelon, P., et al. 2011, A\&A, 529, L6 Ballesteros-Paredes, J., Vázquez-Semadeni, E., Gazol, A., et al. 2011, MNRAS, 416, 1436

Bally, J., Langer, W. D., Stark, A. A., \& Wilson, R. W. 1987, ApJL, 312, L45 Barnes, P. J., Yonekura, Y., Fukui, Y., et al. 2011, ApJS, 196, 12

Battersby, C., \& Bally, J. 2012, in The Labyrinth of Star Formation, ed. D. Stamatellos, S. Goodwin, \& D. Wood-Thompson (Berlin: Springer), in press (arXiv:1208.4608)

Battersby, C., Bally, J., Bernard, J. P., et al. 2011, A\&A, 535, 128

Bertoldi, F., \& McKee, C. 1992, ApJ, 395, 140

Bigiel, F., Leroy, A., Walter, F., et al. 2008, AJ, 136, 2846

Bournaud, F., Elmegreen, B. G., Teyssier, R., Block, D. L., \& Peurari, I. 2010, MNRAS, 409, 1088

Bryan, G. L., Norman, M. L., O'Shea, B. W., et al. 2014, ApJS, 211, 19

Butler, M. J., \& Tan, J. C. 2009, ApJ, 696, 484

Butler, M. J., \& Tan, J. C. 2012, ApJ, 754, 5

Butler, M. J., Tan, J. C., \& Kainulainen, J. 2014, ApJL, 782, L30

Chen, C., \& Ostriker, E. 2014, ApJ, 785, 69

Dobbs, C. 2008, MNRAS, 391, 844

Ferland, G. J., Korista, K. T., Verner, D. A., et al. 1998, PASP, 110, 761
Fiege, J. D., \& Pudritz, R. E. 2000, MNRAS, 311, 85

Ginsburg, A., Bressert, E., Bally, J., \& Battersby, C. 2012, ApJL, 758, L29 Gomez, G. C., \& Vázquez-Semadeni, E. 2014, ApJ, 791, 124

Gutermuth, R. A., Pipher, J. L., Megeath, S. T., et al. 2011, ApJ, 739, 84 Heiderman, A., Evans, N. J., Allen, L. E., Huard, T., \& Heyer, M. 2010, ApJ, 723, 1019

Heitsch, F., Ballesteros-Paredes, J., \& Hartmann, L. 2009, ApJ, 704, 1735

Henshaw, J. D., Caselli, P., Fontani, F., et al. 2013, MNRAS, 428, 3425

Henshaw, J. D., Caselli, P., Fontani, F., Jiménez-Serra, I., \& Tan, J. C. 2014, MNRAS, 440, 2860

Hernandez, A. K., \& Tan, J. C. 2011, ApJ, 730, 44

Hernandez, A. K., Tan, J. C., Kainulainen, J., et al. 2012, ApJL, 756, L13

Inutsuka, S., \& Miyama, S. M. 1992, ApJ, 388, 392

Jackson, J. M., Finn, S. C., Chambers, E. T., Rathborne, J. M., \& Simon, R. 2010, ApJL, 719, L185

Jackson, J. M., Rathborne, J. M., Shah, R. Y., et al. 2006, ApJS, 163, 145

Kainulainen, J., Beuther, H., Henning, T., \& Plume, R. 2009, A\&A, 508, L35

Kainulainen, J., \& Tan, J. C. 2013, A\&A, 549, A53

Kritsuk, A. G., Norman, M. L., \& Wagner, R. 2011, ApJL, 727, L20

Krumholz, M. R., \& Tan, J. C. 2007, ApJ, 654, 304

Lada, C. J., Lombardi, M., \& Alves, J. F. 2010, ApJ, 724, 687

McKee, C. F., \& Ostriker, J. C. 2007, ARA\&A, 45, 565

Moeckel, N., \& Burkert, A. 2014, ApJ, submitted (arXiv:1402.2614)

Nagasawa, M. 1987, PThPh, 77, 635

O'Shea, B. W., Bryan, G., Bordner, J., et al. 2004, arXiv:0403044

Peretto, N., \& Fuller, G. A. 2009, A\&A, 505, 405

Pillai, T., Kauffmann, J., Tan, J. C., et al. 2015, ApJ, 799, 74

Ragan, S. E., Henning, Th., Tackenberg, J., et al. 2014, A\&A, 568, A73 
Rathborne, J. M., Jackson, J. M., \& Simon, R. 2006, ApJ, 641, 389

Renaud, F., Bournaud, F., Emsellem, E., et al. 2013, MNRAS, 436, 1836

Sarazin, C. L., \& White, R. E. 1987, ApJ, 320, 32

Schneider, N., Ossenkopf, V., Csengeri, T., et al. 2014, A\&A, submitted (arXiv:1403.2996)

Smith, R., Glover, S. C. O., \& Klessen, R. S. 2014, MNRAS, 445, 2900

Stodolkiewicz, J. S. 1963, AcA, 13, 30
Stone, J. M., \& Norman, M. L. 1992, ApJS, 80, 753

Tasker, E. J., \& Tan, J. C. 2009, ApJ, 700, 358

Tan, J. C., Kong, S., Butler, M. J., Caselli, P., \& Fontani, F. 2013, ApJ, 779, 96

Truelove, J. K., Klein, R. I., McKee, C. F., et al. 1997, ApJL, 489, L179

Van Loo, S., Butler, M. J., \& Tan, J. C. 2013, ApJ, 764, 36

Van Loo, S., Tan, J. C., \& Falle, S. A. E. G. 2015, ApJ, 800, 11

Zuckerman, B., \& Evans, II, N. J. 1974, ApJL, 192, L149 